\title{
QUANTUM RELATIVISTIC TODA CHAIN AT ROOT OF UNITY: ISOSPECTRALITY, MODIFIED $Q$-OPERATOR, AND FUNCTIONAL BETHE ANSATZ
}

\author{
STANISLAV PAKULIAK and SERGEI SERGEEV
}

Received 17 May 2001 and in revised form 9 April 2002

\begin{abstract}
We investigate an $N$-state spin model called quantum relativistic Toda chain and based on the unitary finite-dimensional representations of the Weyl algebra with $q$ being $N$ th primitive root of unity. Parameters of the finite-dimensional representation of the local Weyl algebra form the classical discrete integrable system. Nontrivial dynamics of the classical counterpart correspond to isospectral transformations of the spin system. Similarity operators are constructed with the help of modified Baxter's $Q$-operators. The classical counterpart of the modified $Q$-operator for the initial homogeneous spin chain is a Bäcklund transformation. This transformation creates an extra Hirota-type soliton in a parameterization of the chain structure. Special choice of values of solitonic amplitudes yields a degeneration of spin eigenstates, leading to the quantum separation of variables, or the functional Bethe ansatz. A projector to the separated eigenstates is constructed explicitly as a product of modified $Q$-operators.
\end{abstract}

2000 Mathematics Subject Classification: 82B23.

1. Introduction. One of the indicative examples of integrable models of mathematical physics is the Toda chain. There are an enormous number of papers in the modern literature concerning the Toda chain, classical as well as quantum one. We mention only [26] as an example of the application of the algebraic methods to the quantum Toda chain. One of the modifications of the Toda chain is known as a relativistic Toda chain, classical as well as quantum, see, for example, [11, 14, 18, 19, 32].

The algebra of observables in the quantum relativistic Toda chain is the local Weyl algebra. Due to an ambiguity of centers of the Weyl algebra with arbitrary Weyl's $q$, we may talk about well-defined quantum model only in three cases. The formal first one is the special limit $q \mapsto 1$, corresponding to the usual quantum Toda chain [26]. The second case is the modular dualization of Weyl's algebra, see [5, 6, 8] for details and [10] for the application of the modular concept to the quantum relativistic Toda chain. In this paper, we are going to discuss the third known case-the case of the unitary finite-dimensional representation of the Weyl algebra, arising when Weyl's parameter $q$ is the $N$ th primitive root of unity. Note, besides the finite-dimensional representations at the root of unity, there exist the real infinite-dimensional ones, but this case belongs to the modular double class.

In several integrable models with the unitary representations of the Weyl algebra at the $N$ th root of unity, the $\mathbb{C}$-valued $N$ th powers of Weyl's elements form a classical discrete integrable system. So the parameters of the unitary representation of the 
Weyl algebra form a classical counterpart of the finite-dimensional integrable system (i.e., spin integrable system), see [2, 3, 7, 20, 21, 22, 23, 24] for example. The most important finite-dimensional operators, arising in the spin systems, have functional counterparts, defined as rational mappings in the space of functions of the $N$ th powers of Weyl's elements. So, the finite-dimensional operators are the secondary objects, we have to define first the mapping of the parameters, and then the finite-dimensional operators have to be constructed in the terms of initial and final values of the parameters. Usual finite-dimensional integrable models correspond to the case when the initial and final parameters coincide for all the operators involved. It means the consideration of the trivial classical dynamics. Algebraically, the conditions of the trivialization are the origin of, for example, Baxter's curve for the chiral Potts model [1, 4], or of the spherical triangle parameterization for the Zamolodchikov-Bazhanov-Baxter model [25].

We may hope to achieve the progress in the spin integrable models by including into the consideration a nontrivial classical dynamics. This dynamics will complicate the situation, but sometimes more complicated systems in the mathematics and physics may be solved more easily-proverbial "a problem should be complicated until it becomes trivial" [31].

The quantum relativistic Toda chain at the root of unity is one of the simplest examples of such combined classical/spin system. This model was investigated in the preprints $[15,16,17]$. The present paper is an attempt to present the detailed and clarified exposition of this investigation.

We will use the language of the quantum inverse scattering method. Besides the formulation of the model in the terms of $L$-operators and transfer matrices, it implies the quantum intertwining relation, allowing to construct Baxter's $Q$-operator. Eigenvalues of the transfer matrix and $Q$-operator satisfy the Baxter, or $T Q=Q^{\prime}+Q^{\prime \prime}$ equation. Baxter equation may hardly be solved explicitly for arbitrary $N$ and for finite length of the chain $M$. It is the key relation for the investigation of the model in the thermodynamic limit. We will not investigate the Baxter equation in this paper even in the thermodynamic limit, we will restrict ourselves by a derivation of it.

Since any quantum Toda-type chain is the realm of the functional Bethe ansatz, the problem of eigenstates of the transfer matrix is related to the problem of the eigenstates of off-diagonal element of the monodromy matrix, see, for example, [9, $26,27,28,29]$ for the details. This is true in our case as well. It is also known that Baxter's $Q$-operators in the classical limit are related to the Bäcklund transformations of the classical chains, see, for example, [12, 13, 30].

In the following paragraph we describe in the shortest way the subject of the present paper.

The main feature of the quantum relativistic Toda chain at the root of unity with the nontrivial classical counterpart is that the quantum intertwining relation contains the Darboux transformation (see [30]) for the $N$ th powers of the Weyl elements. So the Bäcklund transformation is the classical counterpart of the finite-dimensional $Q$-operator at the root of unity automatically. Such $Q$-operators we call the modified $Q$-operators, because they do not form the commutative family. Usual $Q$-operators are their particular cases. Modified $Q$-operators solve in general the isospectrality 
problem for the spin chain. Starting from a homogeneous quantum chain, we may create some number of solitons in parameterization of the inhomogeneities using the Bäcklund transformations. The finite-dimensional counterpart of this procedure is a product of the modified $Q$-operators, giving a similarity transformation of the initial homogeneous transfer matrix. The term "soliton" is used here in Hirota's sense: the system of the $N$ th powers of Weyl's elements may be parameterized by $\tau$-functions, obeying a system of discrete equations. A solitonic solution of this system is given by the Hirota-type expressions. Solitonic $\tau$-functions contain extra free complex parameters - the amplitudes of solitonic partial waves. Generally speaking, these parameters are a kind of "times" of the classical model conjugated to the commutative set of classical Hamiltonians. In the other interpretation the amplitudes stand for a point on a Jacobian of a classical spectral curve in its rational limit. Besides the complete solution of the quantum isospectrality problem, these degrees of freedom appeared to be useful in an unusual sense. The amplitudes may be chosen so that one component of a classical Backer-Akhiezer function becomes zero-this is related to the classical separation of variables. The corresponding finite-dimensional similarity operator becomes a projector to an eigenvector of off-diagonal element of the monodromy matrix, that is, the base of the quantum separation of variables. Moreover, since the similarity operator is the product of $Q$-operators, Sklyanin formula [26] with the product of eigenfunctions $Q$ appears in the direct way.

This paper is devoted to the formulation of the combined classical/spin model-the quantum relativistic Toda chain at the Nth root of unity; its classical Darboux transformation/spin intertwining relation; derivation and parameterization of Bäcklund transformation/modified $Q$-operator; derivation of the Baxter equation; and explicit parameterization of the quantum separating operator.

2. Formulation of the model. In this section, we formulate the model called the quantum relativistic Toda chain at the root of unity.

2.1. $L$-operators. Let the chain be formed by $M$ sites with the periodical boundary conditions. $m$ th site of the Toda chain is described by the following local $L$-operator:

$$
\ell_{m}(\lambda) \stackrel{\text { def }}{=}\left(\begin{array}{cc}
1+\frac{\kappa}{\lambda} \mathbf{u}_{m} \mathbf{w}_{m} & -\frac{\omega^{1 / 2}}{\lambda} \mathbf{u}_{m} \\
\mathbf{w}_{m} & 0
\end{array}\right)
$$

where $\lambda$ is the spectral parameter and $\kappa$ is an extra complex parameter, common for all sites, that is, a modulus of eigenstates. Elements $\mathbf{u}_{m}$ and $\mathbf{w}_{m}$ form the ultra-local Weyl algebra,

$$
\mathbf{u}_{m} \cdot \mathbf{w}_{m}=\omega \mathbf{w}_{m} \cdot \mathbf{u}_{m}
$$

and $\mathbf{u}_{m}, \mathbf{w}_{m}$ for different sites commute. Weyl's parameter $\omega$ is the primitive root of unity,

$$
\omega=\mathbf{e}^{2 \pi i / N}, \quad \omega^{1 / 2}=\mathbf{e}^{i \pi / N} .
$$


$N$ is an arbitrary positive integer greater than one and common for all sites. The $N$ th powers of the Weyl elements are centers of the algebra. We will deal with the finitedimensional unitary representation of the Weyl algebra

$$
\mathbf{u}_{m}=u_{m} \mathbf{x}_{m}, \quad \mathbf{w}_{m}=w_{m} \mathbf{z}_{m},
$$

where $u_{m}$ and $w_{m}$ are $\mathbb{C}$-numbers, and

$$
\mathbf{x}_{m}=1 \otimes \cdots \otimes \underbrace{\mathbf{x}}_{\begin{array}{c}
m \text { th } \\
\text { place }
\end{array}} \otimes \cdots \otimes 1, \quad \mathbf{z}_{m}=1 \otimes \cdots \otimes \underbrace{\mathbf{z}}_{\begin{array}{c}
m \text { th } \\
\text { place }
\end{array}} \otimes \cdots \otimes 1 .
$$

A convenient representation of $\mathbf{x}$ and $\mathbf{z}$ in the $N$-dimensional vector space $|\alpha\rangle=$ $|\alpha \bmod N\rangle$ is

$$
\mathbf{x}|\alpha\rangle=|\alpha\rangle \omega^{\alpha}, \quad \mathbf{z}|\alpha\rangle=|\alpha+1\rangle, \quad\langle\alpha \mid \beta\rangle=\delta_{\alpha, \beta}
$$

Thus $\mathbf{x}$ and $\mathbf{z}$ are $N \times N$ dimensional matrices, normalized to the unity $\left(\mathbf{x}^{N}=\mathbf{z}^{N}=1\right)$, and the $N$ th powers of the local Weyl elements are $\mathbb{C}$-numbers

$$
\mathbf{u}_{m}^{N}=u_{m}^{N}, \quad \mathbf{w}_{m}^{N}=w_{m}^{N} .
$$

In general, all $u_{m}, w_{m}$ are different, that is, $u_{m} \neq u_{m^{\prime}}$ and $w_{m} \neq w_{m^{\prime}}$ such chain is the inhomogeneous one.

Variables $u_{m}^{N}$ and $w_{m}^{N}$ form the classical counterpart of the quantum relativistic Toda chain. Define the classical $L$-operator as

$$
L_{m}\left(\lambda^{N}\right) \stackrel{\text { def }}{=}\left(\begin{array}{cc}
1+\frac{\kappa^{N}}{\lambda^{N}} u_{m}^{N} w_{m}^{N} & \frac{u_{m}^{N}}{\lambda^{N}} \\
w_{m}^{N} & 0
\end{array}\right) .
$$

2.2. Transfer matrices and integrability. Ordered product of the quantum $L^{-}$ operators

$$
\hat{\mathbf{t}}(\lambda) \stackrel{\text { def }}{=} \ell_{1}(\lambda) \ell_{2}(\lambda) \cdots \ell_{M}(\lambda)=\left(\begin{array}{ll}
\mathbf{a}(\lambda) & \mathbf{b}(\lambda) \\
\mathbf{c}(\lambda) & \mathbf{d}(\lambda)
\end{array}\right),
$$

and its trace

$$
\mathbf{t}(\lambda)=\mathbf{a}(\lambda)+\mathbf{d}(\lambda)=\sum_{k=0}^{M} \lambda^{-k} \mathbf{t}_{k}
$$

are the monodromy and the transfer matrices of the quantum model.

The integrability of the quantum chain is provided by the intertwining relation in the auxiliary two-dimensional spaces

$$
R(\lambda, \mu) \ell(\lambda) \otimes \ell(\mu)=(1 \otimes \ell(\mu))(\ell(\lambda) \otimes 1) R(\lambda, \mu) .
$$

The tensor product of two $2 \times 2$ matrices with the identical Weyl algebra entries $\mathbf{u}, \mathbf{w}$, $\kappa$ but different spectral parameters $\lambda$ and $\mu$ is implied in (2.11), and

$$
R(\lambda, \mu)=\left(\begin{array}{cccc}
\lambda-\omega \mu & 0 & 0 & 0 \\
0 & \lambda-\mu & \mu(1-\omega) & 0 \\
0 & \lambda(1-\omega) & \omega(\lambda-\mu) & 0 \\
0 & 0 & 0 & \lambda-\omega \mu
\end{array}\right)
$$


is the slightly twisted six-vertex trigonometric $R$-matrix, used, for example, in [33]. Equation (2.11) may be verified directly. The repeated use of the intertwining relation (2.11) gives the analogous relation for the monodromy matrices (2.9), leading to the commutativity of the transfer matrices (2.10) with different spectral parameters but with the identical elements $\mathbf{u}_{m}, \mathbf{w}_{m}$, and $\kappa$

$$
\left[\mathbf{t}\left(\lambda, \kappa ;\left\{u_{m}, w_{m}\right\}\right), \mathbf{t}\left(\mu, \kappa ;\left\{u_{m}, w_{m}\right\}\right)\right]=0,
$$

where we emphasize the dependence of $\mathbf{t}(\lambda)=\mathbf{t}\left(\lambda, \kappa ;\left\{u_{m}, w_{m}\right\}_{m=1}^{M}\right)$ on the given set of $\mathbb{C}$-valued parameters $u_{m}, w_{m}, m=1, \ldots, M$.

In the spectral decomposition of the transfer matrix (2.10) the utmost operators are

$$
\mathbf{t}_{0}=1, \quad \mathbf{t}_{M}=\kappa^{M} \prod_{m=1}^{M}\left(-\omega^{1 / 2} u_{m} w_{m}\right) \mathbf{Y}
$$

where

$$
\mathbf{Y}=\prod_{m=1}^{M}\left(-\omega^{-1 / 2} \mathbf{x}_{m} \mathbf{z}_{m}\right), \quad \mathbf{Y}^{N}=1 .
$$

Transfer matrix (2.10) is invariant with respect to a gauge transformation of $L$-operators (2.1). Let $G$ be $2 \times 2$ matrix with $\mathbb{C}$-valued coefficients, then the transformation

$$
\ell_{m} \longmapsto G \ell_{m} G^{-1} \Longrightarrow \hat{\mathbf{t}} \longmapsto G \hat{\mathbf{t}} G^{-1}
$$

does not change the transfer matrix. For example,

$$
G=\left(\begin{array}{ll}
1 & 0 \\
0 & g
\end{array}\right)
$$

produces the transformation of the parameters

$$
u_{m} \longmapsto g^{-1} u_{m}, \quad w_{m} \longmapsto g w_{m} .
$$

Taking into account this freedom and also the possibility of redefinition of the spectral parameter $\lambda$, entering $L$-operator (2.1) always as $\lambda^{-1} u_{m}$, we may impose, without loss of generality, the following conditions for the parameters $\left\{u_{m}, w_{m}\right\}_{m=1}^{M}$

$$
\prod_{m=1}^{M}\left(-\omega^{1 / 2} u_{m}\right)=\prod_{m=1}^{M}\left(-w_{m}\right)=1 .
$$

2.3. Classical monodromy matrix. Define the monodromy of the classical Lax matrices (2.8) analogously to (2.9) as

$$
\hat{T} \stackrel{\text { def }}{=} L_{1} L_{2} \cdots L_{M}=\left(\begin{array}{ll}
A\left(\lambda^{N}\right) & B\left(\lambda^{N}\right) \\
C\left(\lambda^{N}\right) & D\left(\lambda^{N}\right)
\end{array}\right) .
$$

For our purposes we have to mention the relation between the elements of the quantum monodromy matrix (2.9) and the classical ones. Note at first, relation (2.11) provides the commutativity

$$
[\mathbf{a}(\lambda), \mathbf{a}(\mu)]=[\mathbf{b}(\lambda), \mathbf{b}(\mu)]=[\mathbf{c}(\lambda), \mathbf{c}(\mu)]=[\mathbf{d}(\lambda), \mathbf{d}(\mu)]=0 .
$$


The spectra of $\mathbf{a}(\lambda), \mathbf{b}(\lambda), \mathbf{c}(\lambda)$, and $\mathbf{d}(\lambda)$ may be calculated by means of the following proposition.

Proposition 2.1. Cyclic products of quantum monodromy matrix elements are the centers:

$$
\begin{array}{ll}
\prod_{n \in \mathbb{Z}_{N}} \mathbf{a}\left(\omega^{n} \lambda\right)=A\left(\lambda^{N}\right), & \prod_{n \in \mathbb{Z}_{N}} \mathbf{b}\left(\omega^{n} \lambda\right)=B\left(\lambda^{N}\right), \\
\prod_{n \in \mathbb{Z}_{N}} \mathbf{c}\left(\omega^{n} \lambda\right)=C\left(\lambda^{N}\right), & \prod_{n \in \mathbb{Z}_{N}} \mathbf{d}\left(\omega^{n} \lambda\right)=D\left(\lambda^{N}\right) .
\end{array}
$$

Theorems of such kind were proved in [33] for the general case of cyclic representations like (2.6) of the quantum affine algebras. An alternative proof follows from the results of [20, 21, 22, 23, 24].

Consider the homogeneous chain with $u_{m}=u$ and $w_{m}=w$. Due to (2.18) and (2.19), we may fix the parameters of the homogeneous chain as

$$
u_{m}=-\omega^{-1 / 2}, \quad w_{m}=-1 .
$$

Then

$$
\hat{T}=L\left(\lambda^{N}\right)^{M}, \quad L\left(\lambda^{N}\right)=\left(\begin{array}{cc}
1-\frac{\kappa^{N}}{\lambda^{N}} & -\frac{\epsilon}{\lambda^{N}} \\
\epsilon & 0
\end{array}\right),
$$

where $\epsilon=(-1)^{N}$, and the matrix elements of $\hat{T}$ may be calculated with the help of projector decomposition of $L\left(\lambda^{N}\right)$. Namely, let $x_{1}$ and $x_{2}$ are two eigenvalues of $L\left(\lambda^{N}\right)$

$$
x_{1} x_{2}=\frac{1}{\lambda^{N}}, \quad x_{1}+x_{2}=1-\frac{\kappa^{N}}{\lambda^{N}} .
$$

Then $L\left(\lambda^{N}\right)$ may be presented in the form $L\left(\lambda^{N}\right)=D \operatorname{diag}\left(x_{1}, x_{2}\right) D^{-1}$, therefore $\widehat{T}=$ $D \operatorname{diag}\left(x_{1}^{M}, x_{2}^{M}\right) D^{-1}$, and the final answer reads

$$
\hat{T}=\left(\begin{array}{cc}
\frac{x_{1}^{M+1}-x_{2}^{M+1}}{x_{1}-x_{2}} & -\epsilon x_{1} x_{2} \frac{x_{1}^{M}-x_{2}^{M}}{x_{1}-x_{2}} \\
\epsilon \frac{x_{1}^{M}-x_{2}^{M}}{x_{1}-x_{2}} & \frac{x_{1} x_{2}^{M}-x_{2} x_{1}^{M}}{x_{1}-x_{2}}
\end{array}\right) .
$$

Now we may calculate $\left(x_{1}^{M}-x_{2}^{M}\right) /\left(x_{1}-x_{2}\right)$ explicitly as a function of $\lambda^{N}$. First of all, it is an $(M-1)$ th power polynomial with respect to $1 / \lambda^{N}$, so

$$
\frac{x_{1}^{M}-x_{2}^{M}}{x_{1}-x_{2}}=C_{0} \prod_{k=1}^{M-1}\left(1-\frac{\lambda_{k}^{N}}{\lambda^{N}}\right) .
$$

In the limit when $1 / \lambda^{N} \mapsto 0$, we have $x_{1}=1$ and $x_{2}=0$, see (2.25). Therefore $C_{0}=1$. The roots $\lambda^{N}=\lambda_{k}^{N}$ of (2.27) correspond to the case when

$$
x_{1}^{M}=x_{2}^{M}, \quad x_{1} \neq x_{2} \Longrightarrow \frac{x_{2}}{x_{1}}=\mathbf{e}^{2 i \phi_{k}},
$$


where $\phi_{k}, k=1, \ldots, M-1$, belong to the following set $\mathfrak{f}_{M}$ :

$$
\phi_{k} \in \mathfrak{f}_{M} \stackrel{\text { def }}{=}\left\{\frac{\pi}{M}, \frac{2 \pi}{M}, \ldots, \frac{(M-1) \pi}{M}\right\} .
$$

The ordering of $\phi_{k} \in \mathfrak{f}_{M}$ is not essential. Introduce now three useful functions $\Delta_{\phi}$, $\Delta_{\phi}^{*}$, and $\Lambda_{\phi}$ as follows:

$$
\begin{aligned}
& \Delta_{\phi}=\mathbf{e}^{i \phi}\left(\sqrt{\cos ^{2} \phi+\kappa^{N}}+\cos \phi\right), \\
& \Delta_{\phi}^{*}=\mathbf{e}^{-i \phi}\left(\sqrt{\cos ^{2} \phi+\kappa^{N}}+\cos \phi\right), \\
& \Lambda_{\phi}=\Delta_{\phi} \Delta_{\phi}^{*} .
\end{aligned}
$$

Expressions for $\Delta$ and $\Delta^{*}$ uniformize the rational curve

$$
\Delta \Delta^{*}=\Delta+\Delta^{*}+\kappa^{N} \text { in the terms of } \frac{\Delta}{\Delta^{*}}=\mathbf{e}^{2 i \phi} .
$$

Comparing (2.25) and (2.31), we conclude

$$
\lambda_{k}^{N}=\Lambda_{\phi_{k}}, \quad \phi_{k} \in \mathfrak{f}_{M} .
$$

The application of Proposition 2.1 to the homogeneous chain gives in particular

$$
\prod_{n \in \mathbb{Z}_{N}} \mathbf{b}\left(\omega^{n} \lambda\right)=-\frac{\epsilon}{\lambda^{N}} \prod_{k=1}^{M-1}\left(1-\frac{\Lambda_{\phi_{k}}}{\lambda^{N}}\right), \quad \phi_{k} \in \mathfrak{f}_{M} .
$$

2.4. Eigenvalues and eigenstates. The natural aim of the investigation of any integrable quantum chain is to calculate the spectrum of the transfer matrix and to construct its eigenstates at least for the homogeneous chain. In this subsection we will fix several notations necessary for the spectral problems and for the functional Bethe ansatz.

For given transfer matrix $\mathbf{t}(\lambda)$, let $t(\lambda)$ be its eigenvalue for the right eigenvector $\left|\Psi_{t}\right\rangle$ and for the left eigenvector $\left\langle\Psi_{t}\right|$,

$$
\mathbf{t}(\lambda)\left|\Psi_{t}\right\rangle=\left|\Psi_{t}\right\rangle t(\lambda), \quad\left\langle\Psi_{t}\right| \mathbf{t}(\lambda)=t(\lambda)\left\langle\Psi_{t}\right| .
$$

Here $t(\lambda)=\sum_{k=0}^{M} \lambda^{-k} t_{k}, t_{k}$ are eigenvalues of the complete commutative set of $\mathbf{t}_{k}(2.10)$. Due to (2.14) and (2.15) $t_{0} \equiv 1$ and $t_{M}=(-\kappa)^{M} \omega^{\gamma}$, where $\omega^{\gamma}$ is the eigenvalue of Y. The subscript " $t$ " in the notations of the eigenvectors stands for the $M$-components vector $\left\{t_{k}\right\}, k=1, \ldots, M$. The eigenvectors do not depend on $\lambda$, but depend on $\kappa$ and $u_{m}, w_{m}$ for the inhomogeneous chain. In general $\mathbf{t}_{k}$ are not Hermitian, so left and right eigenvectors are not conjugated. Nevertheless, imply that $\left|\Psi_{t}\right\rangle$ and $\left\langle\Psi_{t}\right|$ are dual complete bases,

$$
\mathbf{t}(\lambda)=\sum_{t}\left|\Psi_{t}\right\rangle t(\lambda)\left\langle\Psi_{t}\left|, \quad 1=\sum_{t}\right| \Psi_{t}\right\rangle\left\langle\Psi_{t}\right|
$$

where the summations over $t$ is by definition the summation over all $N^{M}$ possible sets of the eigenvalues $\left\{t_{k}\right\}_{k=1}^{M}$. 
All the observables are defined in the local basis initially (2.5)

$$
|\alpha\rangle=\left|\alpha_{1}\right\rangle \otimes\left|\alpha_{2}\right\rangle \otimes \cdots \otimes\left|\alpha_{M}\right\rangle
$$

The dual basis is defined via

$$
\langle\alpha \mid \beta\rangle=\prod_{m=1}^{M} \delta_{\alpha_{m}, \beta_{m}}, \quad 1=\sum_{\left\{\alpha_{m}\right\}}|\alpha\rangle\langle\alpha| .
$$

Calculation of the eigenvector $\left|\Psi_{t}\right\rangle$ means the calculation of the matrix elements $\left\langle\alpha \mid \Psi_{t}\right\rangle$

$$
\left|\Psi_{t}\right\rangle=\sum_{\left\{\alpha_{m}\right\}}|\alpha\rangle\left\langle\alpha \mid \Psi_{t}\right\rangle
$$

Now formulate the isospectrality problem. Let $\left\{u_{m}, w_{m}\right\}_{m=1}^{M}$ and $\left\{u_{m}^{\prime}, w_{m}^{\prime}\right\}_{m=1}^{M}$ be two sets of parameters of Weyl elements for the quantum relativistic Toda chain at the root of unity. These sets are isospectral if

$$
t\left(\lambda ;\left\{u_{m}, w_{m}\right\}_{m=1}^{M}\right)=t\left(\lambda ;\left\{u_{m}^{\prime}, w_{m}^{\prime}\right\}_{m=1}^{M}\right) .
$$

Let $\left|\Psi_{t}\right\rangle$ and $\left|\Psi_{t}^{\prime}\right\rangle$ be the eigenvectors of two isospectral transfer matrices $\mathbf{t}(\lambda)$ and $\mathbf{t}^{\prime}(\lambda)$ with the same eigenvalue $t(\lambda)$. Two isospectral transfer matrices must be related by a similarity operator $\mathbf{K}$

$$
\mathbf{t}\left(\lambda ;\left\{u_{m}, w_{m}\right\}_{m=1}^{M}\right) \mathbf{K}=\mathbf{K t}\left(\lambda ;\left\{u_{m}^{\prime}, w_{m}^{\prime}\right\}_{m=1}^{M}\right),
$$

such that

$$
\mathbf{K}=\sum_{t}\left|\Psi_{t}\right\rangle K_{t}\left\langle\Psi_{t}^{\prime}\right|
$$

Here $K_{t}$ are arbitrary nonzero values. We will prove further that two transfer matrices are isospectral if and only if the traces of their classical monodromy matrices coincide.

The eigenvalues of the transfer matrix obey several functional relations. Sometimes they may be solved in the thermodynamic limit or at least some physical properties of the chain may be derived from the functional equations in the thermodynamic limit.

The eigenstates for quantum Toda chain, relativistic as well as usual, and in general the eigenstates for models based on the local Weyl algebra at root of the unity may be constructed with the help of so called functional Bethe ansatz, or quantum separation of variables [9, 26, 27, 28, 29]. Eigenstates of off-diagonal element of the monodromy matrix $\mathbf{b}(\lambda)$ (2.9) play the important role in the method of the functional Bethe ansatz. It is useful to parameterize the spectrum of $\mathbf{b}(\lambda)$ by its zeros $\lambda_{k}, k=1, \ldots, M-1$. It follows from (2.33) for the homogeneous chain

$$
\lambda_{k}^{N}=\Lambda_{\phi_{k}}, \quad k=1, \ldots, M-1, \phi_{k} \in \mathfrak{f}_{M} .
$$

The monodromy matrix (2.9) has the structure

$$
\hat{\mathbf{t}}(\lambda)=\hat{\mathbf{t}}^{\prime}(\lambda) \cdot \ell_{M}(\lambda),
$$


where $\hat{\mathbf{t}}^{\prime}(\lambda)$ is the monodromy of the first $M-1$ sites. Then

$$
\mathbf{b}(\lambda)=\mathbf{a}^{\prime}(\lambda)\left(-\frac{\omega^{1 / 2}}{\lambda} \mathbf{u}_{M}\right)
$$

It is more convenient to work with the eigenvectors of the operator

$$
\tilde{\mathbf{b}}(\lambda) \stackrel{\text { def }}{=} \mathbf{b}(\lambda) \mathbf{w}_{M}=\mathbf{a}^{\prime}(\lambda)\left(-\frac{\omega^{1 / 2}}{\lambda} \mathbf{u}_{M} \mathbf{w}_{M}\right)
$$

Note that operator $\mathbf{Y}$ in (2.15) commute with $\tilde{\mathbf{b}}$. We denote the eigenvectors of $\tilde{\mathbf{b}}$ as $\left|\left\{\lambda_{k}\right\}_{k=1}^{M-1}, \gamma\right\rangle$ and define them by the following relation:

$$
\begin{gathered}
\mathbf{b}\left(\lambda_{j}\right)\left|\left\{\lambda_{k}\right\}_{k=1}^{M-1}, \gamma\right\rangle=0, \quad \lambda_{j} \in\left\{\lambda_{k}\right\}_{k=1}^{M-1}, \\
\mathbf{Y}\left|\left\{\lambda_{k}\right\}_{k=1}^{M-1}, \gamma\right\rangle=\left|\left\{\lambda_{k}\right\}_{k=1}^{M-1}, \gamma\right\rangle \omega^{\gamma} .
\end{gathered}
$$

The functional Bethe ansatz method, being applied to the quantum relativistic Toda chain at root of unity, implies the following structure of the matrix element (see [27, $28,29])$ :

$$
\left\langle\Psi_{t} \mid\left\{\lambda_{k}\right\}_{k=1}^{M-1}, \gamma\right\rangle=\mathrm{const} \prod_{k=1}^{M-1} q_{t}\left(\lambda_{k}\right) .
$$

$q_{t}(\lambda)$ is a function, which depends on the spectrum of $\mathbf{t}(\lambda)$, and obeys a functional equation, called the Baxter $T-Q$ equation. Operator $\mathbf{Y}$, being the member of $\mathbf{t},(2.14)$, has the eigenvalue $\omega^{\gamma}$ in all the components of formula (2.47). The explicit form of Baxter equation and the definition of $q_{t}(\lambda)$ will be given later.

We obtain usually formula (2.47) from (2.11), see [9, 26, 27, 28, 29]. In this paper, we will obtain (2.47) in a different way: we will construct first the so-called modified $\mathbf{Q}$-operator, whose spectrum is related to $q_{t}$. Then a projector to $\left|\left\{\lambda_{k}\right\}_{k=1}^{M-1}, \gamma\right\rangle$ will be obtained using these modified $\mathbf{Q}$-operators.

3. Intertwining relations. The quantum relativistic Toda chain $L$-operator (2.1) is $2 \times 2$ matrix, whose matrix elements are $N \times N$ matrices. According to the conventional terminology, the two-dimensional vector space is called the auxiliary space, while the $N$-dimensional one is called the quantum space. Equation (2.11) is the intertwining relation in the auxiliary spaces. In this section, we will investigate the other type of the intertwining relations-in the quantum spaces.

It is easy to check that two $L$-operators, (2.1), cannot be intertwined in the quantum spaces (i.e., their intertwiner is exactly zero). In the case of the usual quantum Toda chain we need an extra auxiliary $L$-operator (in its simplest form it is known as the $L$-operator for the "dimer self-trapping model" [30]). The same situation holds in our case, as we need an auxiliary $L$-operator that may be intertwined with $L$-operator (2.1) in the quantum spaces. In general, this auxiliary $L$-operator is Bazhanov-Stroganov $L$-operator for the six-vertex model at the root of unity [4]. This fact is the origin of a relationship between the relativistic Toda chain at the $N$ th root of unity and the $N$-state chiral Potts model. The Bazhanov-Stroganov L-operator may be simplified to a "relativistic dimer self-trapping" $L$-operator. 
In this section, we introduce this auxiliary $L$-operator and write down the quantum intertwining relation. Being written in an appropriate way, this will give us a key for all further investigations.

3.1. Auxiliary $L$-operator. Define the auxiliary quantum $L$-operator as follows:

$$
\tilde{\ell}_{\phi}\left(\lambda, \lambda_{\phi}\right) \stackrel{\text { def }}{=}\left(\begin{array}{cc}
1-\omega^{1 / 2} \kappa_{\phi} \frac{\lambda_{\phi}}{\lambda} \mathbf{w}_{\phi} & -\frac{\omega^{1 / 2}}{\lambda}\left(1-\omega^{1 / 2} \kappa_{\phi} \mathbf{w}_{\phi}\right) \mathbf{u}_{\phi} \\
-\omega^{1 / 2} \lambda_{\phi} \mathbf{u}_{\phi}^{-1} \mathbf{w}_{\phi} & \mathbf{w}_{\phi}
\end{array}\right) .
$$

Here $\lambda$ and $\lambda_{\phi}$ are two spectral parameters (actually, up to a gauge transformation (2.16), $\tilde{\ell}$ depends on their ratio). $\kappa_{\phi}$ is a module analogous to $\kappa . \mathbf{u}_{\phi}$ and $\mathbf{w}_{\phi}$ are additional Weyl elements, defined analogously to (2.2), (2.4), (2.5), and (2.6)

$$
\mathbf{u}_{\phi}=u_{\phi} \mathbf{x}_{\phi}, \quad \mathbf{w}_{\phi}=w_{\phi} \mathbf{z}_{\phi} .
$$

In these notations the subscript $\phi$ labels the additional Weyl pair in the tensor product (2.5).

Classical counterpart of $\tilde{\ell}$ is by definition

$$
\tilde{L}_{\phi}\left(\lambda^{N}, \lambda_{\phi}^{N}\right) \stackrel{\text { def }}{=}\left(\begin{array}{cc}
1+\kappa_{\phi}^{N} \frac{\lambda_{\phi}^{N}}{\lambda^{N}} w_{\phi}^{N} & \frac{u_{\phi}^{N}}{\lambda^{N}}\left(1+\kappa_{\phi}^{N} w_{\phi}^{N}\right) \\
\lambda_{\phi}^{N} \frac{w_{\phi}^{N}}{u_{\phi}^{N}} & w_{\phi}^{N}
\end{array}\right) .
$$

$L$-operators (3.1) are intertwined in their two-dimensional auxiliary vector spaces by the same six-vertex trigonometric $R$-matrix (2.12). There exists also the fundamental quantum intertwiner for (3.1). It is the $R$-matrix for the chiral Potts model such that two rapidities are fixed to special singular values. The details are useless in this paper. But the method described here may be applied directly to the model, defined by (3.1).

3.2. Quantum intertwining relation. We are going to write out some quantum intertwining relation for $L$-operators (2.1) as well as for the whole monodromy matrix (2.9). So we use notations, applicable for the recursion in $m$. Also in this section, we will point out parameters $u_{m}, w_{m}$ and $u_{\phi}, w_{\phi}$ as the arguments of $\ell_{m}$ and $\tilde{\ell}_{\phi}$.

Proposition 3.1. There exists unique (up to a constant multiplier) $N^{2} \times N^{2}$ matrix $\mathbf{R}_{m, \phi}\left(\lambda_{\phi}\right)$, such that $\mathbf{R}_{m, \phi}, \ell_{m}$, and $\tilde{\ell}$ obey the modified intertwining relation

$$
\begin{aligned}
\tilde{\ell}_{\phi}\left(\lambda, \lambda_{\phi} ; u_{\phi, m}, w_{\phi, m}\right) \cdot \ell_{m}\left(\lambda ; u_{m}, w_{m}\right) \mathbf{R}_{m, \phi}\left(\lambda_{\phi}\right) \\
\quad=\mathbf{R}_{m, \phi}\left(\lambda_{\phi}\right) \ell_{m}\left(\lambda ; u_{m}^{\prime}, w_{m}^{\prime}\right) \cdot \tilde{\ell}_{\phi}\left(\lambda, \lambda_{\phi} ; u_{\phi, m+1}, w_{\phi, m+1}\right),
\end{aligned}
$$

if and only if their classical counterparts of $L_{m}$ and $\widetilde{L}_{\phi}$ obey the following Darboux relation:

$$
\begin{aligned}
\tilde{L}_{\phi}\left(\lambda^{N}, \lambda_{\phi}^{N} ; u_{\phi, m}^{N}, w_{\phi, m}^{N}\right) \cdot L_{m}\left(\lambda^{N} ; u_{m}^{N}, w_{m}^{N}\right) \\
\quad=L_{m}\left(\lambda^{N} ; u_{m}^{\prime N}, w_{m}^{\prime N}\right) \cdot \widetilde{L}_{\phi}\left(\lambda^{N}, \lambda_{\phi}^{N} ; u_{\phi, m+1}^{N}, w_{\phi, m+1}^{N}\right),
\end{aligned}
$$

valid in each order of $\lambda^{N}$. 
Proof. Consider the following equation:

$$
\tilde{\ell}_{\phi}\left(\lambda ; \mathbf{u}_{\phi}, \mathbf{w}_{\phi}\right) \cdot \ell_{m}\left(\lambda ; \mathbf{u}_{m}, \mathbf{w}_{m}\right)=\ell_{m}\left(\lambda ; \mathbf{u}_{m}^{\prime}, \mathbf{w}_{m}^{\prime}\right) \cdot \tilde{\ell}_{\phi}\left(\lambda ; \mathbf{u}_{\phi}^{\prime}, \mathbf{w}_{\phi}^{\prime}\right),
$$

and equate its coefficients in each order of $\lambda$. In (3.6) we used the Weyl elements as the formal arguments of $L$-operators (2.1) and (3.1). This system of equations has the unique solution with respect to the primed operators

$$
\begin{aligned}
\mathbf{u}_{m}^{\prime} & =\frac{\kappa_{\phi}}{\kappa} \mathbf{u}_{\phi}, \\
\mathbf{w}_{m}^{\prime} & =\mathbf{w}_{m} \mathbf{w}_{\phi}-\omega^{1 / 2} \lambda_{\phi} \mathbf{u}_{\phi}^{-1} \mathbf{w}_{\phi}, \\
\mathbf{u}_{\phi}^{\prime} & =\left(\lambda_{\phi}+\kappa \mathbf{u}_{m} \mathbf{w}_{m}-\omega^{1 / 2} \mathbf{w}_{m} \mathbf{u}_{\phi}\right)^{-1} \lambda_{\phi} \mathbf{u}_{m}, \\
\mathbf{w}_{\phi}^{\prime} & =\frac{\kappa}{\kappa_{\phi}} \mathbf{u}_{m} \mathbf{w}_{m}\left(\mathbf{w}_{m} \mathbf{u}_{\phi}-\omega^{1 / 2} \lambda_{\phi}\right)^{-1} .
\end{aligned}
$$

Obtaining (3.7) from (3.6), we used the exchange relations for $\mathbf{u}_{m}, \mathbf{w}_{m}, \mathbf{u}_{\phi}$, and $\mathbf{w}_{\phi}$ without assuming any exchange relations for primed operators. We may check directly that the rational homomorphism

$$
\mathbf{u}_{m}, \mathbf{w}_{m}, \mathbf{u}_{\phi}, \mathbf{w}_{\phi} \longmapsto \mathbf{u}_{m}^{\prime}, \mathbf{w}_{m}^{\prime}, \mathbf{u}_{\phi}^{\prime}, \mathbf{w}_{\phi}^{\prime},
$$

given by (3.7), is the automorphism of the local Weyl algebra.

Since the finite-dimensional representations at root of unity are considered (see (2.4) and (3.2)), we may use the normalization of the operators entering (3.6) as usual

$$
\begin{array}{ccc}
\mathbf{u}_{m}=u_{m} \mathbf{x}_{m}, & \mathbf{w}_{m}=w_{m} \mathbf{z}_{m}, \quad \mathbf{u}_{\phi}=u_{\phi, m} \mathbf{x}_{\phi}, & \mathbf{w}_{\phi}=w_{\phi, m} \mathbf{z}_{\phi}, \\
\mathbf{u}_{m}^{\prime}=u_{m}^{\prime} \mathbf{x}_{m}^{\prime}, & \mathbf{w}_{m}^{\prime}=w_{m}^{\prime} \mathbf{z}_{m}^{\prime}, \quad \mathbf{u}_{\phi}^{\prime}=u_{\phi, m+1} \mathbf{x}_{\phi}^{\prime}, & \mathbf{w}_{\phi}^{\prime}=w_{\phi, m+1} \mathbf{z}_{\phi}^{\prime},
\end{array}
$$

where all $\mathbf{x}, \mathbf{z}$ matrices are normalized to unity. With this normalization, the $N$ th powers of (3.7) may be calculated directly with the help of the following identity:

$$
(\mathbf{u}+\mathbf{w})^{N}=\mathbf{u}^{N}+\mathbf{w}^{N} .
$$

The answer reads

$$
\begin{gathered}
u_{\phi, m+1}^{N}=\frac{\lambda_{\phi}^{N} u_{m}^{N}}{\lambda_{\phi}^{N}+\kappa^{N} u_{m}^{N} w_{m}^{N}+w_{m}^{N} u_{\phi, m}^{N}}, \quad w_{\phi, m+1}^{N}=\frac{\kappa^{N}}{\kappa_{\phi}^{N}} \frac{u_{m}^{N} w_{m}^{N}}{\lambda_{\phi}^{N}+w_{m}^{N} u_{\phi, m}^{N}}, \\
u_{m}^{\prime N}=\frac{\kappa_{\phi}^{N}}{\kappa^{N}} u_{\phi, m}^{N}, \quad w_{m}^{\prime N}=\frac{\kappa^{N}}{\kappa_{\phi}^{N}} \frac{u_{m}^{N} w_{m}^{N}}{u_{\phi, m}^{N}} \frac{w_{\phi, m}^{N}}{w_{\phi, m+1}^{N}} .
\end{gathered}
$$

It is easy to check that (3.11) and (3.12) are the exact and the unique solution of (3.5).

Automorphism (3.8) with normalizations (3.9) taken into account, gives the automorphism

$$
\mathbf{x}_{m}, \mathbf{z}_{m}, \mathbf{x}_{\phi}, \mathbf{z}_{\phi} \longmapsto \mathbf{x}_{m}^{\prime}, \mathbf{z}_{m}^{\prime}, \mathbf{x}_{\phi}^{\prime}, \mathbf{z}_{\phi}^{\prime},
$$

where, recall, all $\mathbf{x}, \mathbf{z}$ are normalized to unity finite-dimensional operators. This is provided by (3.11) and (3.12), or by (3.5). Therefore this automorphism must be the 
internal one, that is, due to the Shur lemma there must exist a unique (up to a multiplier) $N^{2} \times N^{2}$ matrix $\mathbf{R}_{m, \phi}$

$$
\begin{array}{rlrl}
\mathbf{x}_{m}^{\prime} & =\mathbf{R}_{m, \phi} \mathbf{x}_{m} \mathbf{R}_{m, \phi}^{-1}, & & \mathbf{z}_{m}^{\prime}=\mathbf{R}_{m, \phi} \mathbf{z}_{m} \mathbf{R}_{m, \phi}^{-1}, \\
\mathbf{x}_{\phi}^{\prime}=\mathbf{R}_{m, \phi} \mathbf{x}_{\phi} \mathbf{R}_{m, \phi}^{-1}, & \mathbf{z}_{\phi}^{\prime}=\mathbf{R}_{m, \phi} \mathbf{z}_{\phi} \mathbf{R}_{m, \phi}^{-1} .
\end{array}
$$

The last two equations are equivalent to (3.4).

The local transformation

$$
u_{m}^{N}, w_{m}^{N}, u_{\phi, m}^{N}, w_{\phi, m}^{N} \longmapsto u_{m}^{N}, w_{m}^{\prime N}, u_{\phi, m+1}^{N}, w_{\phi, m+1}^{N},
$$

given by (3.11) and (3.12), is called the Darboux transformation for the classical relativistic Toda chain, see, for example, [30] for the analogous transformation for the usual Toda chain. Equations (3.11) and (3.12) define the mapping (3.15) up to $N$ th roots of unity. These phases are the additional discrete parameters appeared when we take the $N$ th roots in (3.15). Note, the matrix $\mathbf{R}_{m, \phi}$ is unique if all these roots are fixed.

In the next section we will give matrix elements of $\mathbf{R}_{m, \phi}$ in the basis (2.6).

3.3. 2-transformation and the isospectrality problem. Relations (3.4) and (3.5) may be iterated for the whole chain. The functional counterpart, (3.5), gives

$$
\widetilde{L}_{\phi}\left(u_{\phi, 1}^{N}, w_{\phi, 1}^{N}\right) \hat{T}\left(\left\{u_{m}^{N}, w_{m}^{N}\right\}_{m=1}^{M}\right)=\hat{T}\left(\left\{u_{m}^{\prime N}, w_{m}^{\prime N}\right\}_{m=1}^{M}\right) \widetilde{L}_{\phi}\left(u_{\phi, M+1}^{N}, w_{\phi, M+1}^{N}\right),
$$

where $\hat{T}$ is the classical monodromy matrix (2.20) and the spectral parameters are implied. Values of $u_{m}^{\prime}, w_{m}^{\prime}, m=1, \ldots, M$, and $u_{\phi, M+1}, w_{\phi, M+1}$ in the terms of $\lambda_{\phi}, \kappa_{\phi}$, $u_{m}, w_{m}$, and $u_{\phi, 1}, w_{\phi, 1}$ must be obtained as the recursion iterating (3.11) and (3.12) for $m=1, \ldots, M$.

For the periodic chain, the cyclic boundary conditions for the recursion have to be imposed,

$$
u_{\phi, M+1}=u_{\phi, 1}, \quad w_{\phi, M+1}=w_{\phi, 1} .
$$

Now suppose that (3.11) and (3.17) are solved, that is, $u_{\phi, m}, w_{\phi, m}$ are parameterized (at least implicitly) in the terms of $u_{m}, w_{m}, m=1, \ldots, M$, and some extra parameters, possible degrees of freedom of (3.11) and (3.17) (e.g., $\lambda_{\phi}, \kappa_{\phi}$, etc.). Then (3.12) defines in general the transformation $2_{\phi}$

$$
2_{\phi}:\left\{u_{m}, w_{m}\right\}_{m=1}^{M} \longmapsto\left\{u_{m}^{\prime}, w_{m}^{\prime}\right\}_{m=1}^{M} .
$$

The transfer matrices for two sets $\left\{u_{m}, w_{m}\right\}$ and $\left\{u_{m}^{\prime}, w_{m}^{\prime}\right\}$ have the same spectrum, because of the existence of $N^{M} \times N^{M}$ matrix $\mathbf{Q}_{\phi}$, nondegenerative in general,

$$
\mathbf{Q}_{\phi} \stackrel{\text { def }}{=} \operatorname{tr}_{\phi} \hat{\mathbf{Q}}_{\phi}, \quad \hat{\mathbf{Q}}_{\phi} \stackrel{\text { def }}{=} \mathbf{R}_{1, \phi} \mathbf{R}_{2, \phi} \cdots \mathbf{R}_{M, \phi},
$$

such that

$$
\mathbf{t}\left(\lambda ;\left\{u_{m}, w_{m}\right\}\right) \cdot \mathbf{Q}_{\phi}=\mathbf{Q}_{\phi} \cdot \mathbf{t}\left(\lambda ;\left\{u_{m}^{\prime}, w_{m}^{\prime}\right\}\right) .
$$


The subscript $\phi$ in $\mathbf{Q}_{\phi}$ stands as the reminder for the parameters, arising in the solution of recursion, including at least the spectral parameter $\lambda_{\phi}$, so $\mathbf{Q}_{\phi}$ means in particular $\mathbf{Q}\left(\lambda_{\phi}\right)$. It is important to point out once more that $\mathbf{Q}_{\phi}$ remembers about its initial $\left\{u_{m}, w_{m}\right\}_{m=1}^{M}$ and its final $\left\{u_{m}^{\prime}, w_{m}^{\prime}\right\}_{m=1}^{M}$ sets of the parameters. In terms of the eigenvectors of the transfer matrix $\mathbf{t}(\lambda)$ (2.34), the operator $\mathbf{Q}_{\phi}$ can be written as

$$
\mathbf{Q}_{\phi}=\sum_{t}\left|\Psi_{t}\right\rangle q_{t, \phi}\left\langle\Psi_{t}^{\prime}\right|
$$

Consider now the repeated application of the transformations $2_{\phi},(3.18)$,

$$
\left\{u_{m}, w_{m}\right\} \stackrel{\mathscr{Q}_{\phi_{1}}}{\longmapsto}\left\{u_{m}^{\prime}, w_{m}^{\prime}\right\} \longmapsto \cdots\left\{u_{m}^{(n-1)}, w_{m}^{(n-1)}\right\} \stackrel{\mathscr{I}_{\phi_{n}}}{\longmapsto}\left\{u_{m}^{(n)}, w_{m}^{(n)}\right\} \longmapsto \cdots\left\{u_{m}^{(g)}, w_{m}^{(g)}\right\},
$$

such that the set of isospectral quantum transfer matrices

$$
\mathbf{t}^{(n)}(\lambda)=\mathbf{t}\left(\lambda, \kappa ;\left\{u_{m}^{(n)}, w_{m}^{(n)}\right\}_{m=1}^{M}\right), \quad n=0, \ldots, g
$$

has arisen. The sequence (3.22) defines the transformation $\mathscr{K}$,

$$
\mathscr{K}^{(g)}:\left\{u_{m} \equiv u_{m}^{(0)}, w_{m} \equiv w_{m}^{(0)}\right\}_{m=1}^{M} \longmapsto\left\{u_{m}^{(g)}, w_{m}^{(g)}\right\}_{m=1}^{M},
$$

with the finite-dimensional counterpart

$$
\mathbf{K}^{(g)}=\mathbf{Q}_{\phi_{1}}^{(1)} \mathbf{Q}_{\phi_{2}}^{(2)} \cdots \mathbf{Q}_{\phi_{n}}^{(n)} \cdots \mathbf{Q}_{\phi_{g}}^{(g)},
$$

where

$$
\mathbf{Q}_{\phi_{n}}^{(n)}=\operatorname{tr}_{\phi_{n}}\left(\mathbf{R}_{1, \phi_{n}}^{(n)} \mathbf{R}_{2, \phi_{n}}^{(n)} \cdots \mathbf{R}_{M, \phi_{n}}^{(n)}\right),
$$

such that

$$
\mathbf{t}^{(n-1)}(\lambda) \mathbf{Q}_{\phi_{n}}^{(n)}=\mathbf{Q}_{\phi_{n}}^{(n)} \mathbf{t}^{(n)}(\lambda), \quad \mathbf{t}^{(0)}(\lambda) \mathbf{K}^{(g)}=\mathbf{K}^{(g)} \mathbf{t}^{(g)}(\lambda) .
$$

Thus in general the transformations $\mathscr{2}_{\phi}$ and their iterations $\mathscr{K}^{(g)}$ give the isospectral transformations of the initial quantum chain in the space of the parameters, so that their finite-dimensional counterparts $\mathbf{Q}_{\phi}$ and $\mathbf{K}^{(g)}$ respectfully describe the change of the eigenvector basis (see (2.41)).

4. Matrix R. In this section, we construct explicitly the finite-dimensional matrix $\mathbf{R}_{m, \phi}$, obeying (3.4). Since a basis invariant formula for $\mathbf{R}_{m, \phi}$ is useless and rather complicated, we will find matrix elements of $\mathbf{R}_{m, \phi}$ in the basis (2.6). But first we have to introduce several notations concerning the functions on the Fermat curve.

4.1. $w$-function. Let $p$ be a point on the Fermat curve $\mathscr{F}$

$$
p \stackrel{\text { def }}{=}(x, y) \in \mathscr{F} \Longleftrightarrow x^{N}+y^{N}=1 .
$$

Actually, identity (3.10) is the origin of the Fermat curve. Very useful function on the Fermat curve is $w_{p}(n), p \in \mathscr{F}, n \in \mathbb{Z}_{N}$, defined as follows:

$$
\frac{w_{p}(n)}{w_{p}(n-1)}=\frac{y}{1-x \omega^{n}}, \quad w_{p}(0)=1 .
$$


The function $w_{p}(n)$ has a lot of remarkable properties, see the appendix of [25] for an introduction to $\omega$-hypergeometry. In this paper, it is necessary to mention just a couple of properties of $w$-function. Let $O$ be the following automorphism of the Fermat curve:

$$
p=(x, y) \Longleftrightarrow O p=\left(\omega^{-1} x^{-1}, \omega^{-1 / 2} x^{-1} y\right)
$$

Then

$$
w_{p}(n) w_{O p}(-n) \Phi(n)=1 \text {, where } \Phi(n)=(-)^{n} \omega^{n^{2} / 2} .
$$

In the subsequent sections we will use also two simple properties of $w$-function,

$$
w_{(x, \omega y)}(n)=\omega^{n} w_{(x, y)}(n), \quad w_{(x, y)}(n+1)=\frac{y}{1-\omega x} w_{(\omega x, y)}(n) .
$$

Define also three special points on the Fermat curve

$$
q_{0}=(0,1), \quad q_{\infty}=O q_{0}, \quad q_{1}=\left(\omega^{-1}, 0\right) .
$$

Then

$$
w_{q_{0}}(n)=1, \quad w_{q_{\infty}}(n)=\frac{1}{\Phi(n)}, \quad \frac{1}{w_{q_{1}}(n)}=\delta_{n, 0} .
$$

The inversion relation may be mentioned for the completeness

$$
\sum_{n \in \mathbb{Z}_{M}} \frac{w_{(\omega x, \omega y)}(n+a)}{w_{(x, y)}(n+b)}=N \frac{1-\omega x}{\omega x} \frac{x^{N}}{1-x^{N}} \delta_{a, b} .
$$

4.2. Matrix elements of $\mathbf{R}_{m, \phi}$. Consider the $N^{2} \times N^{2}$ matrix $\mathbf{R}_{m, \phi}\left(p_{1}, p_{2}, p_{3}\right)$ with the following matrix elements:

$$
\left\langle\alpha_{m}, \alpha_{\phi}\left|\mathbf{R}_{m, \phi}\right| \beta_{m}, \beta_{\phi}\right\rangle=\omega^{\left(\alpha_{m}-\beta_{m}\right) \beta_{\phi}} \frac{w_{p_{1}}\left(\alpha_{\phi}-\alpha_{m}\right) w_{p_{2}}\left(\beta_{\phi}-\beta_{m}\right)}{w_{p_{3}}\left(\beta_{\phi}-\alpha_{m}\right)} \delta_{\alpha_{\phi}, \beta_{m}} .
$$

Here $p_{1}, p_{2}$, and $p_{3}$ are three points on the Fermat curve, such that

$$
x_{1} x_{2}=x_{3}
$$

Equation (4.10) and the spin structure of (4.9) provide the dependence of (4.9) on two continuous parameters, say $x_{1}$ and $x_{3}$, and on two discrete parameters, say the phase of $y_{1}$ and the phase of $y_{3}$.

Proposition 4.1. The matrix $\mathbf{R}_{m, \phi}\left(p_{1}, p_{2}, p_{3}\right)$, whose matrix elements (4.9) are given in the basis (2.6), makes the following mapping:

$$
\begin{aligned}
\mathbf{R}_{m, \phi} \mathbf{x}_{m} \mathbf{R}_{m, \phi}^{-1} & =\mathbf{x}_{\phi}, \\
\mathbf{R}_{m, \phi} \mathbf{z}_{m} \mathbf{R}_{m, \phi}^{-1} & =\frac{y_{3}}{y_{2}} \mathbf{z}_{m} \mathbf{z}_{\phi}-\omega \frac{x_{3} y_{1}}{x_{1} y_{2}} \mathbf{x}_{\phi}^{-1} \mathbf{z}_{\phi}, \\
\mathbf{R}_{m, \phi} \mathbf{x}_{\phi}^{-1} \mathbf{R}_{m, \phi}^{-1} & =\omega x_{3} \mathbf{x}_{m}^{-1}-\omega \frac{x_{1} y_{3}}{y_{1}} \mathbf{x}_{m}^{-1} \mathbf{z}_{m} \mathbf{x}_{\phi}+\frac{y_{3}}{y_{1}} \mathbf{z}_{m}, \\
\mathbf{R}_{m, \phi} \mathbf{z}_{\phi}^{-1} \mathbf{R}_{m, \phi}^{-1} & =\frac{y_{3}}{y_{2}} \mathbf{x}_{\phi} \mathbf{x}_{m}^{-1}-\omega \frac{x_{3} y_{1}}{x_{1} y_{2}} \mathbf{z}_{m}^{-1} \mathbf{x}_{m}^{-1}
\end{aligned}
$$


PROOF. Each relation of (4.11) should be rewritten in the form

$$
\left\langle\alpha_{m}, \alpha_{\phi}\left|\mathbf{R}_{m, \phi} \mathbf{f}=\mathbf{f}^{\prime} \mathbf{R}_{m, \phi}\right| \beta_{m}, \beta_{\phi}\right\rangle .
$$

Such identities may be verified directly with the help of (4.5).

Compare (4.11) with (3.7). The matrix $\mathbf{R}_{m, \phi}$ solves (3.4) if

$$
\begin{gathered}
x_{1}=\omega^{-1 / 2} \frac{u_{\phi, m}}{\kappa u_{m}}, \quad \frac{x_{3} y_{1}}{x_{1} y_{3}}=\omega^{-1 / 2} \frac{\lambda_{\phi}}{u_{\phi, m} w_{m}}, \\
u_{m}^{\prime}=\frac{\kappa_{\phi}}{\kappa} u_{\phi, m}, \quad w_{m}^{\prime}=w_{m} w_{\phi, m} \frac{y_{2}}{y_{3}}, \\
u_{\phi, m+1}=\omega x_{3} u_{m}, \quad w_{\phi, m+1}=\frac{\kappa}{\kappa_{\phi}} \frac{u_{m}}{u_{\phi, m}} \frac{y_{3}}{y_{2}} .
\end{gathered}
$$

Equations (4.13) and (4.14) are the complete set of the relations following from the identification of (4.11) with (3.7). Using (4.13), (4.10), and (4.1), we have to fix first the parameters $p_{1}, p_{2}, p_{3}$ and thus to define $\mathbf{R}_{m, \phi}$. Then (4.14) parameterize $u_{m}^{\prime}, w_{m}^{\prime}$, $u_{\phi, m+1}, w_{\phi, m+1}$ in the terms of $u_{m}, w_{m}, u_{\phi, m}, w_{\phi, m}$ and the phases of $x_{3}$ and $y_{2} / y_{3}$.

When we consider the complicated operator $\mathbf{K}^{(g)}$, (3.25), its matrix elements must be calculated using matrices $\mathbf{R}_{m, \phi_{n}}^{(n)}$, entering (3.26). The matrix elements of all $\mathbf{R}_{m, \phi_{n}}^{(n)}$ are the same functions,

$$
\mathbf{R}_{m, \phi_{n}}^{(n)}=\mathbf{R}_{m, \phi_{n}}\left(p_{1, m}^{(n)}, p_{2, m}^{(n)}, p_{3, m}^{(n)}\right)
$$

given by (4.9), but with different and rather complicated parameters. Equations (4.13) and (4.14) are written for $p_{1, m}^{(1)}, p_{2, m}^{(1)}, p_{3, m}^{(1)}$ in fact. Nevertheless, for given $\mathscr{K}^{(g)}$, that is, for given sets of $\left\{u_{m}^{(n)}, w_{m}^{(n)}\right\}, m=1, \ldots, M$ and $n=0, \ldots, g$, see (3.22), we may parameterize the corresponding $\mathbf{K}^{(g)}$ explicitly via parameterizations (4.13) and (4.14), the form of the matrix elements of $\mathbf{R}_{m, \phi}$ (4.9), and formulas (3.26) and (3.25).

4.3. Normalization of the matrix R. The matrix elements of $\mathbf{R}_{m, \phi}$ are defined in general up to a constant multiplier. Definition (4.2) and formula (4.9) are the most simple expressions in the terms of the Fermat curve coordinates $p_{1}, p_{2}, p_{3}$. But this $\mathbf{R}_{m, \phi}$ is not normalized. For several applications we need the normalization of $\mathbf{R}_{m, \phi}$ connected with the determinant of $\mathbf{R}_{m, \phi}$.

To calculate the determinant, we need several definitions. Let

$$
V(x) \stackrel{\text { def }}{=} \prod_{n=1}^{N-1}\left(1-\omega^{n+1} x\right)^{n},
$$

$V(x)$ obeys the following relations:

$$
\begin{gathered}
\frac{V\left(\omega^{-1} x\right)}{V(x)}=\frac{1-x^{N}}{(1-x)^{N}} \\
V\left(\omega^{-1} x^{-1}\right)=\frac{\left(1-x^{N}\right)^{N-1} \omega^{N(N-1)(2 N-1) / 6}}{(-x)^{N(N-1) / 2} V(x)} .
\end{gathered}
$$


Besides, we may calculate the particular value

$$
V\left(\omega^{-1}\right) \equiv N^{N / 2} \mathbf{e}^{i \pi(N-1)(N-2) / 12} .
$$

Function $V(x)$ appears in the following expressions, where $p=(x, y)$ :

$$
\begin{gathered}
\prod_{n} \sum_{\sigma} \frac{\omega^{n \sigma}}{w_{p}(\sigma)}=(\omega x)^{N(N-1) / 2} \frac{V\left(\omega^{-1}\right)}{V(x)}, \\
\prod_{n} \sum_{\sigma} \frac{\omega^{n \sigma}}{\Phi(\sigma) w_{p}(\sigma)}=\frac{V\left(\omega^{-1}\right)}{V(x)}, \\
\prod_{n} w_{p}(n)=\frac{V(x)}{y^{N(N-1) / 2}} .
\end{gathered}
$$

The third expression is rather trivial. We may prove the first two formulas considering the poles and zeros of the left- and right-hand sides. Details may be found in the appendix of [25].

Proposition 4.2. The determinant of the matrix $\mathbf{R}$, defined by (4.9), is

$$
\operatorname{det} \mathbf{R}=(-)^{N(N-1) / 2}\left(\prod_{n} \frac{w_{p_{2}}(n)}{w_{O p_{1}}(n)}\right)^{N}\left(\prod_{n} \sum_{\sigma} \frac{\omega^{n \sigma}}{\Phi(\sigma) w_{p_{3}}(\sigma)}\right)^{N} .
$$

Proof. To obtain (4.20), we have to use $O$-automorphism (4.4) for $w_{p_{1}}$ in (4.9). The factors $w_{p_{2}}$ and $w_{O p_{1}}$ correspond to the diagonal matrices $D$ and $D^{\prime}$ in the matrix decomposition of $\mathbf{R}_{m, \phi}$,

$$
\mathbf{R}_{m, \phi}=D\left(p_{1}\right) R^{\prime}\left(p_{3}\right) D^{\prime}\left(p_{2}\right) .
$$

The determinants of $D$ and $D^{\prime}$ give the term with $p_{1}$ and $p_{2}$ in (4.20). The Fourier transform applied to the matrix $\mathbf{R}_{m, \phi}$ in the $m$ th space yields $F_{m} R^{\prime}\left(p_{3}\right) F_{m}^{-1}=D^{\prime \prime}\left(p_{3}\right) P_{m, \phi}$, where $\left\langle\alpha_{m}|F| \beta_{m}\right\rangle=N^{-1 / 2} \omega^{\alpha_{m} \beta_{m}}, D^{\prime \prime}$ is a diagonal matrix and $P_{m, \phi}$ is the permutation. Determinant $\operatorname{det} D^{\prime \prime}$ gives the term, depending on $p_{3}$ in (4.20), and $\operatorname{det} P$ is the sign factor in (4.20).

Let the normalization factor for $\mathbf{R}_{m, \phi}$ be $\rho_{R}$,

$$
\rho_{R}^{N}=\left(\omega^{-1 / 2} \frac{y_{1}}{x_{1} y_{2}}\right)^{N(N-1) / 2} \frac{V\left(x_{1}\right)}{V\left(\omega^{-1} x_{2}^{-1}\right) V\left(x_{3}\right)},
$$

so that

$$
\operatorname{det} \mathbf{R}=\text { const } \cdot \rho_{R}^{N^{2}}
$$

Analogously, the normalization factor for the monodromy of $\mathbf{R}_{m, \phi}$ and so of $\mathbf{Q}_{\phi}$ is

$$
\rho_{Q}^{N}=\prod_{m=1}^{M}\left(\omega^{-1 / 2} \frac{y_{1, m}}{x_{1, m} y_{2, m}}\right)^{N(N-1) / 2} \frac{V\left(x_{1, m}\right)}{V\left(\omega^{-1} x_{2, m}^{-1}\right) V\left(x_{3, m}\right)} .
$$


5. Parameterization of the recursion. The main object of the present investigations is the system of the recursion relations (3.11). Our goal is the construction of operator $\mathbf{K}^{(g)}$, (3.25), for the homogeneous initial state (5.1). In this case, the first step transformation $2_{\phi_{1}}$ has a remarkably simple structure, clarifying nevertheless the structure of all subsequent $2_{\phi_{n}}$.

5.1. The first step. Let the initial parameters $u_{m}, w_{m}$ are homogeneous

$$
u_{m}=-w^{-1 / 2}, \quad w_{m}=-1
$$

see (2.23). The main recursion relation is the first equation in (3.11). For the homogeneous initial state it contains only one unknown $u_{\phi, m}$. Without loss of generality, introduce complex numbers $\delta_{\phi}$ and $\delta_{\phi}^{*}$ ( $*$ does not stand for the complex conjugation!) and a function $\tau_{m}^{\prime}, m \in \mathbb{Z}$, such that

$$
u_{\phi, m}=-\omega^{-1 / 2} \delta_{\phi} \frac{\tau_{m-1}^{\prime}}{\tau_{m}^{\prime}}, \quad \delta_{\phi}^{*}=\frac{\lambda_{\phi}}{\delta_{\phi}} .
$$

The first relation in (3.11) may be rewritten as the second order linear recursion for $\left(\tau_{m}^{\prime}\right)^{N}$ :

$$
\delta_{\phi}^{N} \delta_{\phi}^{* N}\left(\tau_{m}^{\prime}\right)^{N}=\delta_{\phi}^{N}\left(\tau_{m-1}^{\prime}\right)^{N}+\delta_{\phi}^{* N}\left(\tau_{m+1}^{\prime}\right)^{N}+\kappa^{N}\left(\tau_{m}^{\prime}\right)^{N}
$$

Parameterization (5.2) and linear recursion (5.3) are considered now in the general position, without mentioning $\mathbb{Z}_{M}$-invariance. It is known that the general solution of a second order linear recursion with constant coefficients is a linear combination of two fundamental solutions, $\left(\tau_{m}^{\prime}\right)^{N}=\alpha z_{1}^{m}+\beta z_{2}^{m}$, where $z_{1}$ and $z_{2}$ are two roots of the characteristics equation $\left(\delta_{\phi} \delta_{\phi}^{*}\right)^{N}=\delta_{\phi}^{N} z^{-1}+\delta_{\phi}^{* N} z+\kappa^{N}$. We may always redefine $\left(\tau_{m}^{\prime}\right)^{N} \mapsto \alpha^{-1} z_{1}^{-m}\left(\tau_{m}^{\prime}\right)^{N}$. This corresponds to a redefinition of $\delta_{\phi}$. We may choose $\delta_{\phi}$

$$
\delta_{\phi}^{N} \delta_{\phi}^{* N}=\delta_{\phi}^{N}+\delta_{\phi}^{* N}+\kappa^{N}, \quad \lambda_{\phi}=\delta_{\phi} \delta_{\phi}^{*}
$$

such that $z_{1}=1$ and $\tau_{m}^{\prime}=1$ solves (5.3). Compare (5.4) with (2.30) and (2.31). We may identify

$$
\delta_{\phi}^{N}=\Delta_{\phi}, \quad \delta_{\phi}^{* N}=\Delta_{\phi}^{* N}, \quad \lambda_{\phi}^{N}=\Lambda_{\phi},
$$

so the index $\phi$ may be understood conveniently as the spectral parameter $\phi$.

The second fundamental solution of the characteristics equation is $z_{2}=\left(\delta_{\phi} / \delta_{\phi}^{*}\right)^{N}$, that is, $\left(\tau_{m}^{\prime}\right)^{N}=\left(\delta_{\phi}^{N} / \delta_{\phi}^{* N}\right)^{m}$. The complete solution of recursion (5.3) is

$$
\left(\tau_{m}^{\prime}\right)^{N}=1-f_{\phi} \mathbf{e}^{2 i m \phi},
$$

where $f_{\phi}$ is arbitrary complex number, and (see (2.30))

$$
\mathbf{e}^{2 i \phi}=\frac{\delta_{\phi}^{N}}{\delta_{\phi}^{* N}} \equiv \frac{\Delta_{\phi}}{\Delta_{\phi}^{*}} .
$$


Turn now to the $\mathbb{Z}_{M}$-invariance condition. It demands $\tau_{M+m}^{\prime}=\tau_{m}^{\prime}$, hence the possible values of $\phi$ become discrete, $\mathbf{e}^{2 i \phi M}=1$ and $\mathbf{e}^{2 i \phi} \neq 1$. Since there exists the symmetry between $\delta_{\phi}$ and $\delta_{\phi}^{*}$ in (5.3), we may regard $\phi \in \mathfrak{f}_{M}$ in (5.6), see (2.29) for the definition of $\mathfrak{f}_{M}$.

Turn further to the first relation in (3.12) (or to the first relation in (4.14)). It follows that

$$
u_{m}^{\prime}=-\omega^{-1 / 2} \frac{\kappa_{\phi}}{\kappa} \delta_{\phi} \frac{\tau_{m-1}^{\prime}}{\tau_{m}^{\prime}} .
$$

Taking into account the gauge invariance (2.18), we may fix $\kappa_{\phi}$ as follows:

$$
\kappa_{\phi}=\frac{\kappa}{\delta_{\phi}}
$$

and so

$$
u_{m}^{\prime}=-\omega^{-1 / 2} \frac{\tau_{m-1}^{\prime}}{\tau_{m}^{\prime}}
$$

It agrees with (2.19). The second equations in (3.11) and (3.12) give

$$
w_{m}^{\prime}=-\frac{\theta_{m}^{\prime}}{\theta_{m-1}^{\prime}}
$$

where

$$
\left(\theta_{m}^{\prime}\right)^{N}=1-f_{\phi} \frac{\Delta_{\phi}^{*}}{\Delta_{\phi}} \frac{\Delta_{\phi}-1}{\Delta_{\phi}^{*}-1} \mathbf{e}^{2 i m \phi} .
$$

These simple calculations show that with the useful parameterizations (5.5), where the functions of $\phi$ are defined in (2.30), and with additional condition (5.9), the image of $2_{\phi}$-transformation (5.6), (5.10), (5.11), and (5.12) contains one arbitrary complex parameter $f_{\phi}$ if $\phi \in \mathfrak{f}_{M}$. The case when $-\phi \in \mathfrak{f}_{M}$ is equivalent to the case when $\phi \in \mathfrak{f}_{M}$. It follows from the symmetry between $\delta_{\phi}$ and $\delta_{\phi}^{*}$. The case when $\phi$ is arbitrary complex number, $\mathbb{Z}_{M}$-invariance requirement yields $f_{\phi} \equiv 0$, therefore $\tau_{m}^{\prime}=1$ and the chain remains the homogeneous one.

Equations (5.6) and (5.12) define the $N$ th powers of $\tau^{\prime}$ and $\theta^{\prime}$. Their phases are arbitrary. The $N$ th rooting is the subject of the definition of $p_{j, m}$ for $\mathbf{R}_{m, \phi}$.

Functions $\left(\boldsymbol{\tau}_{m}^{\prime}\right)^{N}$ and $\left(\theta_{m}^{\prime}\right)^{N}$ look like solutions of a Hirota-type discrete equation. We call these expressions one-soliton $\tau$-functions. Therefore $2_{\phi}$-transformation with $\phi \in \mathfrak{f}_{M}$ must be identified with the Bäcklund transformation for the classical relativistic Toda chain, see [30] for the application of this ideology to the usual Toda chain.

5.2. Nth step. Turn now to sequence (3.22) of $2_{\phi_{n}}$-transformations

$$
2_{\phi_{n}}:\left\{u_{m}^{(n-1)}, w_{m}^{(n-1)}\right\}_{m=1}^{M} \longmapsto\left\{u_{m}^{(n)}, w_{m}^{(n)}\right\}_{m=1}^{M}
$$

with generic $\lambda_{\phi_{n}}$, parameterized according to (5.5) and (2.30) as follows:

$$
\begin{array}{cc}
\delta_{n}^{N}=\Delta_{n}, \quad \delta_{n}^{* N}=\Delta_{n}^{*}, & \lambda_{\phi_{n}}=\delta_{n} \delta_{n}^{*}, \\
\Delta_{n} \Delta_{n}^{*}=\Delta_{n}+\Delta_{n}^{*}+\kappa^{N}, & \mathrm{e}^{2 i \phi_{n}}=\frac{\Delta_{n}}{\Delta_{n}^{*}} .
\end{array}
$$


Taking into account (2.19), we may parameterize the $n$th state in sequence (3.22), $n=0, \ldots, g$, as

$$
u_{m}^{(n)}=-\omega^{-1 / 2} \frac{\tau_{m-1}^{(n)}}{\tau_{m}^{(n)}}, \quad w_{m}^{(n)}=-\frac{\theta_{m}^{(n)}}{\theta_{m-1}^{(n)}}, \quad m \in \mathbb{Z}_{M}
$$

The homogeneous initial state (2.23) corresponds to $\tau_{m}^{(0)}=\theta_{m}^{(0)}=1$. Parameterizations (5.15) with the first relation of (3.12) taken into account, implies that

$$
\begin{gathered}
\kappa_{\phi_{n}}=\frac{\kappa}{\delta_{n}}, \\
u_{\phi_{n}, m}=-\omega^{-1 / 2} \delta_{n} \frac{\tau_{m-1}^{(n)}}{\tau_{m}^{(n)}}
\end{gathered}
$$

are imposed for $2_{\phi_{n}}$-transformation. The key recursion relation, the first one from (3.11), looks now as follows:

$$
\begin{aligned}
\left(\delta_{\phi_{n}} \delta_{\phi_{n}}^{*} \boldsymbol{\tau}_{m}^{(n)} \boldsymbol{\tau}_{m}^{(n-1)} \theta_{m-1}^{(n-1)}\right)^{N}= & \left(\delta_{\phi_{n}} \boldsymbol{\tau}_{m-1}^{(n)} \boldsymbol{\tau}_{m}^{(n-1)} \theta_{m}^{(n-1)}\right)^{N} \\
& +\left(\delta_{\phi_{n}}^{*} \boldsymbol{\tau}_{m+1}^{(n)} \boldsymbol{\tau}_{m-1}^{(n-1)} \theta_{m-1}^{(n-1)}\right)^{N}+\left(\kappa \boldsymbol{\tau}_{m}^{(n)} \boldsymbol{\tau}_{m-1}^{(n-1)} \theta_{m}^{(n-1)}\right)^{N}
\end{aligned}
$$

The second equations in (3.11) and (3.12) provide

$$
\begin{gathered}
w_{\phi_{n}, m+1}=w_{\phi_{n}} \frac{\theta_{m}^{(n-1)} \tau_{m}^{(n)}}{\tau_{m}^{(n-1)} \theta_{m}^{(n)}}, \quad w_{\phi_{n}}^{N}=\frac{1}{1-\Delta_{\phi_{n}}^{*}}, \\
\left(\theta_{m}^{(n)}\right)^{N}=\left(\frac{w_{\phi_{n}}}{\tau_{m-1}^{(n-1)}}\right)^{N}\left(\left(\theta_{m}^{(n-1)} \tau_{m-1}^{(n)}\right)^{N}-\left(\delta_{\phi_{n}}^{*} \theta_{m-1}^{(n-1)} \tau_{m}^{(n)}\right)^{N}\right) .
\end{gathered}
$$

Consider (5.18) and (5.20) as the set of discrete equations with respect to $m \in \mathbb{Z}$ and $n \in \mathbb{Z}_{+}$with the initial data $\tau_{m}^{(0)}=\theta_{m}^{(0)}=1$.

Proposition 5.1. General solution of the infinite-dimensional in $m$ and in $n$ system of discrete equations (5.18) and (5.20) with generic values of $\phi_{n}, n=0,1, \ldots$ (see parameterizations (5.14)) and with the initial data $\tau_{m}^{(0)}=\theta_{m}^{(0)}=1$ is given by

$$
\begin{aligned}
& \left(\tau_{m}^{(n)}\right)^{N}=h^{(n)}\left(\left\{f_{k} \mathbf{e}^{2 i m \phi_{k}}\right\}_{k=1}^{n}\right), \\
& \left(\theta_{m}^{(n)}\right)^{N}=h^{(n)}\left(\left\{f_{k} s_{k}(1) \mathbf{e}^{2 i m \phi_{k}}\right\}_{k=1}^{n}\right),
\end{aligned}
$$

where $f_{1}, \ldots, f_{n} \equiv\left\{f_{k}\right\}_{k=1}^{n}$ is a set of arbitrary complex variables, $h^{(n)}$ are defined by

$$
h^{(1)}\left(f_{1}\right)=1-f_{1},
$$

and recursively

$$
h^{(n)}\left(\left\{f_{k}\right\}_{k=1}^{n}\right)=h^{(n-1)}\left(\left\{f_{k} s_{k}\left(\Delta_{n}^{*}\right)\right\}_{k=1}^{n-1}\right)-f_{n} \prod_{m=1}^{n-1} s_{n}\left(\Delta_{m}^{*}\right) h^{(n-1)}\left(\left\{f_{k} s_{k}\left(\Delta_{n}\right)\right\}_{k=1}^{n-1}\right),
$$

where the functions $s_{n}$ are

$$
s_{n}(\xi)=\frac{\Delta_{n}^{*}}{\Delta_{n}} \frac{\Delta_{n}-\xi}{\Delta_{n}^{*}-\xi} .
$$


Proof. Equations (5.18) and (5.20) become the algebraic identities after the substitution (5.21) (see Proposition .5 in the appendix). The first step of this recursion with respect to $n$ is described before, hence $\left(\tau_{m}^{\prime}\right)^{N}=h^{(1)}\left(f \mathrm{e}^{2 i \phi m}\right)$, and so forth .

Suppose that the statement of the proposition is true for $n=1, \ldots, g-1$. The function $h^{(g)}$ depends on $f_{1}, \ldots, f_{g}$, while $h^{(g-1)}$ depends only on $f_{1}, \ldots, f_{g-1}$, therefore $\tau_{m}^{(g)}$ contains one extra degree of freedom $f_{g} \in \mathbb{C}$ with respect to $\tau_{m}^{(g-1)}, \theta_{m}^{(g-1)}$. On the other side, (5.18) with $n=g$ is the second order linear recursion with respect to $\left(\tau_{m}^{(g)}\right)^{N}$, so it must have exactly two fundamental solutions. Hence, due to the homogeneity, general normalized solution of (5.18) with $n=g$ with respect to $\left(\tau_{m}^{(g)}\right)^{N}$ for given $\tau_{m}^{(g-1)}, \theta_{m}^{(g-1)}$ must have exactly one extra degree of freedom. This degree of the freedom is parameter $f_{g}$.

Proposition 5.1 is formulated for the generic sequence of $\phi_{n}$. We may verify, due to definition (5.23), $h^{(g)}\left(\left\{f_{n}\right\}_{n=1}^{g}\right)$ is the symmetrical function with respect to any permutation of the pairs $\left(f_{n}, \phi_{n}\right), n=1, \ldots, g$.

Two special cases should be discussed. The fist one is the case when $\phi_{n}=\phi_{n^{\prime}} \bmod \pi$ for some $n$ and $n^{\prime}$, and the second one is $\phi_{n}=-\phi_{n}^{\prime} \bmod \pi$. Both these cases are the singular ones for definition (5.23). Nevertheless, since the observables $u_{m}^{(g)}$ and $w_{m}^{(g)}$ are the ratios of $\tau$-functions, corresponding $\tau_{m}^{(g)}$ and $\theta_{m}^{(g)}$ for these cases may be obtained as the residues of formula (5.21). Technically, both cases are equivalent (up to some re-parameterizations of $\kappa_{\phi_{n}}$ or $\kappa_{\phi_{n^{\prime}}}$ ). The equivalence is connected again with the symmetry between $\delta_{\phi}$ and $\delta_{\phi}^{*}$, so we may consider only the case

$$
\phi_{n}=-\phi_{n^{\prime}} \bmod \pi
$$

In this case condition (5.16) is convenient for any $n$, and the residues of $\tau_{m}^{(g)}$ and $\theta_{m}^{(g)}$ are given by the same formula (5.21), but with

$$
f_{n}=f_{n^{\prime}} \equiv 0 \text {. }
$$

Turn now the finite chain with the periodic boundary conditions

$$
\tau_{M+m}^{(g)}=\tau_{m}^{(g)}, \quad \theta_{M+m}^{(g)}=\theta_{m}^{(g)} .
$$

This boundary conditions, being applied to (5.21), give the following rule: in the sequence of $f_{1}, \ldots, f_{g}$

$$
f_{n}=0 \Longleftrightarrow \phi_{n} \notin \mathfrak{f}_{M} \bmod \pi,
$$

where the set $\mathfrak{f}_{M}$ is given by (2.29). It means that any $2_{\phi}$ with $\phi \notin \mathfrak{f}_{M}$ just changes the values of $f_{n}$. Therefore the maximal number of the possible exponents $\mathbf{e}^{2 i m \phi_{n}}$ is $M-1$. Without loss of generality, define the minimal complete sequence of $2_{\phi_{n}}$ described by

$$
\left\{\left(f_{n}, \phi_{n}\right)\right\}_{n=1}^{M-1}: \phi_{n} \in \mathfrak{f}_{M}, \quad \phi_{n} \neq \phi_{n^{\prime}},
$$

such that the corresponding operator $\mathbf{K}^{(M-1)}$ creates the state of the quantum chain with $(M-1)$ arbitrary complex parameters. 
Usually the expressions for $\left(\tau_{m}^{(g)}\right)^{N},\left(\theta_{m}^{(g)}\right)^{N}$, given by (5.21), are called the solitonic solutions of discrete Hirota-type equations. Integer $g$ is the number of solitons, $\mathbf{e}^{2 i m \phi_{n}}$ is $n$th solitonic wave (or exponent) and $f_{n}$ is the amplitude of $n$th partial wave. $2_{\phi_{n}}$ mapping, $\phi_{n} \in \mathfrak{f}_{M}$, increases the number of the solitons, hence it is the Bäcklund transformation.

5.3. Parameterization of modified $Q$-operators. Turn now to the parameterizations of the matrix elements of the finite-dimensional counterpart of the transformation $2_{\phi_{n}}$-the matrix $\mathbf{Q}_{\phi_{n}}$. By definition (3.26), it is constructed with the help of R-matrices, and to parameterize it, we have to point out the Fermat curve parameters (4.15).

All the ingredients are already prepared: one has just substitute the parameterizations (5.15), (5.16), (5.17), and (5.19) into (4.13) and (4.14). As the result, $\mathbf{R}_{m, \phi_{n}}^{(n)}{ }^{-}$ matrices, entering (3.26), have the following arguments (see (4.15)):

$$
\begin{aligned}
& x_{1, m}^{(n)}=\omega^{-1 / 2} \frac{\delta_{n}}{\kappa} \frac{\boldsymbol{T}_{m}^{(n-1)} \boldsymbol{T}_{m-1}^{(n)}}{\boldsymbol{\tau}_{m-1}^{(n-1)} \boldsymbol{T}_{m}^{(n)}}, \quad x_{3, m}^{(n)}=\omega^{-1} \delta_{n} \frac{\boldsymbol{\tau}_{m}^{(n-1)} \boldsymbol{T}_{m}^{(n)}}{\boldsymbol{\tau}_{m-1}^{(n-1)} \boldsymbol{T}_{m+1}^{(n)}}, \\
& \frac{y_{3, m}^{(n)}}{y_{2, m}^{(n)}}=w_{\phi_{n}} \frac{\theta_{m}^{(n-1)} \tau_{m-1}^{(n)}}{\tau_{m-1}^{(n-1)} \theta_{m}^{(n)}}, \quad \frac{y_{1, m}^{(n)}}{y_{3, m}^{(n)}}=\omega^{1 / 2} \frac{\lambda_{n}}{\kappa \delta_{n}} \frac{\theta_{m-1}^{(n-1)} \tau_{m+1}^{(n)}}{\theta_{m}^{(n-1)} \tau_{m}^{(n)}},
\end{aligned}
$$

and due to (4.10)

$$
x_{2, m}^{(n)}=\omega^{-1 / 2} \kappa \frac{\left(\tau_{m}^{(n)}\right)^{2}}{\tau_{m-1}^{(n)} \tau_{m+1}^{(n)}} .
$$

The trace of quantum monodromy (3.26) may be calculated explicitly in the basis (2.6)

$$
\left\langle\alpha\left|\mathbf{Q}_{\phi_{n}}^{(n)}\right| \beta\right\rangle=\prod_{m \in \mathbb{Z}_{M}} \omega^{\left(\alpha_{m}-\beta_{m}\right) \beta_{m+1}} \frac{w_{p_{1, m}^{(n)}}\left(\beta_{m}-\alpha_{m}\right) w_{p_{2, m}^{(n)}}\left(\beta_{m+1}-\beta_{m}\right)}{w_{p_{3, m}^{(n)}}\left(\beta_{m+1}-\alpha_{m}\right)} .
$$

Thus, the operator $\mathbf{K}^{(g)}$, (3.25), calculated as the product of $g Q^{(n)}$-operators, is defined explicitly.

5.4. Permutation of the modified $Q$-operators. For a given state $\left\{u_{m}, w_{m}\right\}_{m=1}^{M}$, consider two successive 2 -transformations (3.18) with different $\phi$-parameters $\phi_{1}$ and $\phi_{2}$. For the simplicity let $\left\{u_{m}, w_{m}\right\}_{m=1}^{M}$ be a solitonic state, described by (5.15) with (5.21). These two transformations may be applied in two different orders, but in our definition of $h^{(g)}(5.23)$ as the symmetrical functions of the pairs $\left(\phi_{k}, f_{k}\right)$ the result should be the same. Of course, the intermediate states are different. These two different ordering are shown in Figure 5.1.

In the terms of the transfer matrices and modified $\mathbf{Q}$-operators the diagram means

$$
\begin{aligned}
& \mathbf{t}\left(\lambda ;\left\{u_{m}, w_{m}\right\}_{m=1}^{M}\right) \mathbf{Q}_{\phi_{1}}=\mathbf{Q}_{\phi_{1}} \mathbf{t}\left(\lambda ;\left\{u_{m}^{\prime}, w_{m}^{\prime}\right\}_{m=1}^{M}\right), \\
& \mathbf{t}\left(\lambda ;\left\{u_{m}^{\prime}, w_{m}^{\prime}\right\}_{m=1}^{M}\right) \mathbf{Q}_{\phi_{2}}=\mathbf{Q}_{\phi_{2}} \mathbf{t}\left(\lambda ;\left\{u_{m}^{\prime \prime}, w_{m}^{\prime \prime}\right\}_{m=1}^{M}\right)
\end{aligned}
$$




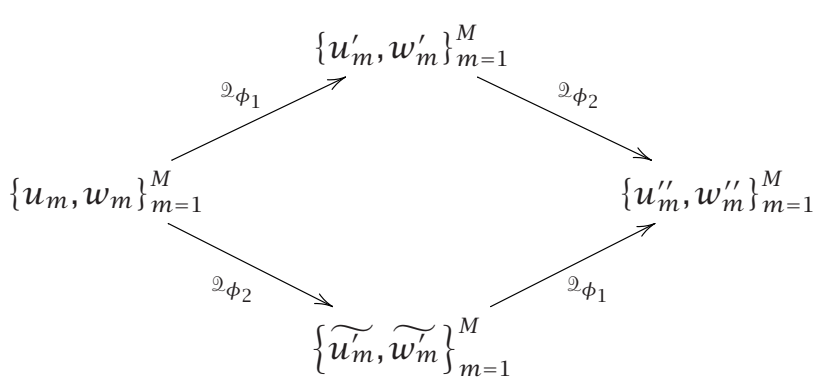

FIGURE 5.1. Two different ordering of $2_{\phi_{1}}$ and $2_{\phi_{2}}$.

versus

$$
\begin{aligned}
& \mathbf{t}\left(\lambda ;\left\{u_{m}, w_{m}\right\}_{m=1}^{M}\right) \widetilde{\mathbf{Q}}_{\phi_{2}}=\widetilde{\mathbf{Q}}_{\phi_{2}} \mathbf{t}\left(\lambda ;\left\{\widetilde{u_{m}^{\prime}}, \widetilde{w_{m}^{\prime}}\right\}_{m=1}^{M}\right), \\
& \mathbf{t}\left(\lambda ;\left\{\widetilde{u_{m}^{\prime}}, \widetilde{w_{m}^{\prime}}\right\}_{m=1}^{M}\right) \widetilde{\mathbf{Q}}_{\phi_{1}}=\widetilde{\mathbf{Q}}_{\phi_{1}} \mathbf{t}\left(\lambda ;\left\{u_{m}^{\prime \prime}, w_{m}^{\prime \prime}\right\}_{m=1}^{M}\right) .
\end{aligned}
$$

Since the result of both mappings is the same, two products of different $\mathbf{Q}$-operators should be the same up to a multiplier. This multiplier corresponds to the normalization factors (4.24)

$$
\rho_{Q_{1}}^{-1} \rho_{Q_{2}}^{-1} \mathbf{Q}_{\phi_{1}} \mathbf{Q}_{\phi_{2}}=\rho_{\widetilde{Q}_{2}}^{-1} \rho_{\widetilde{Q}_{1}}^{-1} \widetilde{\mathbf{Q}}_{\phi_{2}} \widetilde{\mathbf{Q}}_{\phi_{1}} .
$$

This equation is provided actually by an intertwining relation

$$
\rho_{R_{1}}^{-1} \rho_{R_{2}}^{-1} \mathbf{R}_{m, \phi_{1}} \mathbf{R}_{m, \phi_{2}} \mathbf{S}_{\phi_{1}, \phi_{2}}=\rho_{R_{1}^{\prime}}^{-1} \rho_{R_{2}^{\prime}}^{-1} \mathbf{S}_{\phi_{1}, \phi_{2}}^{\prime} \mathbf{R}_{m, \phi_{2}}^{\prime} \mathbf{R}_{m, \phi_{1}}^{\prime},
$$

where $\mathbf{S}$ is a sort of the Chiral Potts model $R$-matrix. These details are not essential in this paper and besides need a lot of extra notations and definitions.

Equation (5.35) provides the following proposition.

Proposition 5.2. Operator $\mathbf{K}^{(M-1)}$, given by (3.25), and parameterized with the help of $\tau_{m}^{(n)}$ and $\theta_{m}^{(n)}$, defined for the minimal complete sequence (5.29), does not depend (up to a multiplier!) on the ordering of the data $\left\{\left(f_{n}, \phi_{n}\right)\right\}_{n=1}^{M-1}$ thus, without loss of generality, we may fix the ordering in (3.25) and in $\mathfrak{f}_{M}$ as follows:

$$
\phi_{n}=\frac{\pi n}{M}, \quad n=1, \ldots, M-1 .
$$

5.5. Inhomogeneous chain. In this section, we investigate and solve the problem of the isospectrality for the homogeneous initial state (2.23) of the quantum chain. For the chain of the size $M$ with the periodic boundary conditions the solution is described in the terms of Hirota-type solitonic $\tau$-functions (5.21) (the number of independent $f_{n}$ is equal to $M-1$ ).

Usual Q-operators are the particular cases of our modified Q-operators. Namely, consider the first step of the recursion in $n$ (5.3) once more. If $\phi$ in (5.6) and (5.12) is such that $\phi \notin \mathfrak{f}_{M} \bmod \pi$ (this corresponds to a generic value of $\lambda_{\phi}$ ) then $\mathbb{Z}_{M}$-periodicity 
demands $f \equiv 0$, and $\tau_{m}^{\prime}=\theta_{m}^{\prime}=1$. It means, $2_{\phi}$-transformation is the trivial one, and the corresponding $\mathbf{Q}_{\phi}$-operator changes nothing. Evidently, such $Q$-operators $\mathbf{Q}\left(\lambda_{\phi}\right)$ commute with the transfer matrix $\mathbf{t}(\lambda)$ and form the commutative family. They are the usual Baxter $Q$-operators.

In general, we may start from the arbitrary initial states, when $\tau_{m}^{(0)}$ and $\theta_{m}^{(0)}$ depend on $m$. Equation (2.13) still provides the integrability of the model. But even on the first step we get the inhomogeneous linear recursion for $\left(\tau_{m}^{\prime}\right)^{N}$, see (5.3). Being considered with $m \in \mathbb{Z}_{M}$, this linear recursion is the null eigenvector problem for $M \times M$ matrix, constructed with the help of $\left(\boldsymbol{\tau}_{m}\right)^{N},\left(\theta_{m}\right)^{N}$, and $\lambda_{\phi}^{N}$. Such equations may be solved if the determinant of this $M \times M$ matrix is zero. This condition defines a hyperelliptic curve $\Gamma_{M-1}$ with the genus $g=M-1$ in general position (see Section 6 for the details). The values of $\left(\tau_{m}^{(n)}\right)^{N}$ and $\left(\theta_{m}^{(n)}\right)^{N}$ may be parameterized in terms of $\theta$-functions on $\operatorname{Jac}\left(\Gamma_{M-1}\right)$. Our expressions (5.23) may be considered as the rational limit of hyperelliptic $\theta$-functions. The most interesting feature of such inhomogeneous models is that we may not construct at all a commutative family of $\mathbf{Q}$-operators.

6. The Baxter equation. In this section, we derive the Baxter $T-Q$ equation in the most general operator form. We will use the well-known method of the triangulization of the auxiliary $L$-operators.

6.1. Generic inhomogeneous chain. Consider the inhomogeneous quantum chain, such that all $u_{m}, w_{m}$ are in general position. Let the normalization (2.19) be implied nevertheless. Turn to the quantum intertwining relation (3.4) and formulas (3.5), (3.11), and (3.12).

The auxiliary $L$-operator (3.1) has the degeneration point at $\lambda=\lambda_{\phi}$

$$
\tilde{\ell}_{\phi}\left(\lambda_{\phi}, \lambda_{\phi}\right)=\left(\begin{array}{c}
1-\omega^{1 / 2} \kappa_{\phi} \mathbf{w}_{\phi} \\
-\omega^{1 / 2} \lambda_{\phi} \mathbf{u}_{\phi}^{-1} \mathbf{w}_{\phi}
\end{array}\right) \cdot\left(1,-\omega^{1 / 2} \lambda_{\phi}^{-1} \mathbf{u}_{\phi}\right)
$$

Analogously, $\tilde{\ell}_{\phi}^{-1}\left(\lambda \mapsto \lambda_{\phi}\right)$ also may be decomposed with the help of two vectors, orthogonal to ones in (6.1). In the degeneration point quantum intertwining relation (3.4) may be rewritten in the vector form. To do this, we will use the row vector in (6.1) and the column vector, orthogonal to it

$$
\psi_{\phi, m}^{\prime *} \stackrel{\text { def }}{=}\left(1,-\omega^{1 / 2} \frac{u_{\phi, m}}{\lambda_{\phi}} \mathbf{x}_{\phi}\right), \quad \psi_{\phi, m}^{\prime \prime} \stackrel{\text { def }}{=}\left(\begin{array}{c}
\omega^{1 / 2} \frac{u_{\phi, m}}{\lambda_{\phi}} \mathbf{x}_{\phi} \\
1
\end{array}\right) .
$$

Here we use the notation $\psi$ for the column vectors with operator-valued entries, and $\psi^{*}$ for the similar row vectors. The following two equations are the triangular form of intertwining relation (3.4) (the dot stands for the matrix multiplication)

$$
\begin{aligned}
& \psi_{\phi, m}^{\prime *} \cdot \ell_{m}\left(\lambda_{\phi} ; u_{m}, w_{m}\right) \mathbf{R}_{m, \phi}=\mathbf{R}_{m, \phi}^{\prime} \psi_{\phi, m+1}^{*}, \\
& \ell_{m}\left(\lambda_{\phi} ; u_{m}^{\prime}, w_{m}^{\prime}\right) \cdot \mathbf{R}_{m, \phi} \psi_{\phi, m+1}^{\prime \prime}=\psi_{\phi, m}^{\prime \prime} \mathbf{R}_{m, \phi}^{\prime \prime}
\end{aligned}
$$


Here

$$
\begin{aligned}
& \mathbf{R}_{m, \phi}^{\prime}=\frac{u_{m}}{u_{\phi, m+1}} \mathbf{x}_{m} \mathbf{R}_{m, \phi} \mathbf{x}_{\phi}^{-1} \\
& \mathbf{R}_{m, \phi}^{\prime \prime}=\omega^{1 / 2} \frac{u_{\phi, m+1} w_{m}}{\lambda_{\phi}} \mathbf{z}_{m} \mathbf{R}_{m, \phi} \mathbf{x}_{\phi}
\end{aligned}
$$

and the recursion relations (3.11) and (3.12) are implied. Equations (6.3) and (6.4) can be verified by the direct calculations in the component form.

Let further $\mathbf{Q}_{\phi}^{\prime}$ and $\mathbf{Q}_{\phi}^{\prime \prime}$ be the traces of the monodromies of $\mathbf{R}_{m, \phi}^{\prime}$ and $\mathbf{R}_{m, \phi}^{\prime \prime}$ similar to (3.19) and we imply the $\mathbb{Z}_{M}$ boundary conditions for the chain and recursion (3.11). For two vectors $\psi_{\phi, 1}^{\prime *}=\psi_{\phi, M+1}^{\prime *}$ and $\psi_{\phi, 1}^{\prime \prime}=\psi_{\phi, M+1}^{\prime \prime}$, let $\psi_{\phi, 1}^{\prime}$ and $\psi_{\phi, 1}^{\prime \prime *}$ be their dual vectors

$$
\psi^{\prime *} \cdot \psi^{\prime}=\psi^{\prime \prime *} \cdot \psi^{\prime \prime}=1, \quad \psi^{\prime *} \cdot \psi^{\prime \prime}=\psi^{\prime \prime *} \cdot \psi^{\prime}=0
$$

such that the $2 \times 2$ unity matrix may be decomposed as follows:

$$
\psi^{\prime} \psi^{\prime *}+\psi^{\prime \prime} \psi^{\prime \prime *}=1
$$

This decomposition allows to calculate

$$
\begin{aligned}
\mathbf{t}\left(\lambda_{\phi}\right) \mathbf{Q}_{\phi} & =\operatorname{tr}_{\mathbb{C}^{2}, \phi}\left(\left(\psi_{\phi, 1}^{\prime} \psi_{\phi, 1}^{\prime *}+\psi_{\phi, 1}^{\prime \prime} \psi_{\phi, 1}^{\prime \prime *}\right) \cdot \hat{\mathbf{t}}\left(\lambda_{\phi}\right) \hat{\mathbf{Q}}_{\phi}\right) \\
& =\operatorname{tr}_{\mathbb{C}^{2}, \phi}\left(\psi_{\phi, 1}^{\prime} \psi_{\phi, 1}^{\prime *} \cdot \hat{\mathbf{t}}\left(\lambda_{\phi}\right) \hat{\mathbf{Q}}_{\phi}+\widehat{\mathbf{t}}\left(\lambda_{\phi}\right) \hat{\mathbf{Q}}_{\phi} \cdot \psi_{\phi, M+1}^{\prime \prime} \psi_{\phi, M+1}^{\prime \prime *}\right) \\
& =\operatorname{tr}_{\phi}\left(\psi_{\phi, 1}^{\prime *} \cdot \hat{\mathbf{Q}}_{\phi} \psi_{\phi, 1}^{\prime}+\psi_{\phi, 1}^{\prime \prime *} \cdot \hat{\mathbf{Q}}_{\phi} \psi_{\phi, 1}^{\prime \prime}\right) \\
& =\mathbf{Q}_{\phi}^{\prime}+\mathbf{Q}_{\phi}^{\prime \prime} .
\end{aligned}
$$

The last equality here is obtained using recursions (6.3). Equation (6.7) gives the Baxter equation in its operator form

$$
\mathbf{t}\left(\lambda_{\phi}\right) \mathbf{Q}_{\phi}=\mathbf{Q}_{\phi} \mathbf{t}^{\prime}\left(\lambda_{\phi}\right)=\mathbf{Q}_{\phi}^{\prime}+\mathbf{Q}_{\phi}^{\prime \prime}
$$

where $\mathbf{t}(\lambda)$ is the initial transfer matrix with the parameters $u_{m}, w_{m}$, and $\mathbf{t}^{\prime}(\lambda)$ is the transfer matrix with the parameters $u_{m}^{\prime}, w_{m}^{\prime}$. Using (6.4) and the first relation of (4.11), we may obtain

$$
\mathbf{Q}_{\phi}^{\prime}=\prod_{m} \frac{u_{m}}{u_{\phi, m}} \mathbf{X Q}_{\phi} \mathbf{X}^{-1}, \quad \mathbf{Q}^{\prime \prime}=\prod_{m} \omega^{1 / 2} \frac{u_{\phi, m} w_{m}}{\lambda_{\phi}} \mathbf{Z Q}_{\phi} \mathbf{X}
$$

where $\mathbf{X}$ and $\mathbf{Z}$ are given by

$$
\mathbf{X}=\prod_{m=1}^{M} \mathbf{x}_{m}, \quad \mathbf{Z}=\prod_{m=1}^{M} \mathbf{z}_{m} .
$$


Note that in the spectral decomposition of $\mathbf{t}(\lambda)$ and $\mathbf{t}^{\prime}(\lambda)$ there exists the operator $\mathbf{Y}$, (2.15). The operator $\mathbf{Q}_{\phi}^{\prime \prime}$ may be rewritten as

$$
\mathbf{Q}_{\phi}^{\prime \prime}=\left(\prod_{m=1}^{M}-\frac{u_{\phi, m} w_{m}}{\lambda_{\phi}}\right) \mathbf{Y X}^{-1} \mathbf{Q}_{\phi} \mathbf{X}
$$

Using (5.32), we may check $\mathbf{Y Q}_{\phi}=\mathbf{Q}_{\phi} \mathbf{Y}$ (the matrix elements should be taken). Let now (see (2.19))

$$
\prod_{m=1}^{M} \frac{u_{\phi, m}}{u_{m}}=\mu_{\phi}, \quad \prod_{m=1}^{M} u_{\phi, m} w_{m}=\omega^{-M / 2} \mu_{\phi}
$$

The Baxter equation in the operator form becomes

$$
\mathbf{t}\left(\lambda_{\phi}\right) \mathbf{Q}_{\phi}=\mathbf{Q}_{\phi} \mathbf{t}^{\prime}\left(\lambda_{\phi}\right)=\mu_{\phi}^{-1} \mathbf{X} \mathbf{Q}_{\phi} \mathbf{X}^{-1}+\frac{\mu_{\phi}}{\left(-\omega^{1 / 2} \lambda_{\phi}\right)^{M}} \mathbf{Y} \mathbf{X}^{-1} \mathbf{Q}_{\phi} \mathbf{X}
$$

The natural question arises: what is $\mu_{\phi}$ ? To answer it, consider (3.5) in the degeneration point $\lambda=\lambda_{\phi}$, applied to the classical monodromy matrix $\hat{T},(2.20)$ and (3.16). Using the formulas like (6.3) for the classical counterpart, we obtain

$$
\begin{gathered}
\left(1, \frac{u_{\phi, 1}^{N}}{\lambda_{\phi}^{N}}\right) \cdot \hat{T}\left(\lambda_{\phi}^{N}\right)=\prod_{m=1}^{M} \frac{u_{m}^{N}}{u_{\phi, m+1}^{N}} \cdot\left(1, \frac{u_{\phi, M+1}^{N}}{\lambda_{\phi}^{N}}\right), \\
\hat{T}\left(\lambda_{\phi}^{N}\right) \cdot\left(\begin{array}{c}
-\frac{u_{\phi, M+1}^{N}}{\lambda_{\phi}^{N}} \\
1
\end{array}\right)=\left(\begin{array}{c}
-\frac{u_{\phi, 1}^{N}}{\lambda_{\phi}^{N}} \\
1
\end{array}\right) \prod_{m=1}^{M}\left(-\frac{u_{\phi, m+1}^{N} w_{m}^{N}}{\lambda_{\phi}^{N}}\right) .
\end{gathered}
$$

When the $\mathbb{Z}_{M}$ boundary condition $u_{\phi, M+1}=u_{\phi, 1}$ is imposed, we may see that both $\mu_{\phi}^{-N}$ and $\mu_{\phi}^{N} /\left(\lambda_{\phi}^{N}\right)^{M}$ are the eigenvalues of the classical monodromy matrix, that is,

$$
J\left(\lambda_{\phi}^{N}, \mu_{\phi}^{N}\right) \stackrel{\text { def }}{=} \operatorname{det}\left(\mu_{\phi}^{-N}-\widehat{T}\left(\lambda_{\phi}^{N}\right)\right)=0
$$

or

$$
A\left(\lambda_{\phi}^{N}\right)+D\left(\lambda_{\phi}^{N}\right)=\frac{1}{\mu_{\phi}^{N}}+\frac{\mu_{\phi}^{N}}{\lambda_{\phi}^{N M}} .
$$

Hence $\left(\lambda_{\phi}^{N}, \mu_{\phi}^{N}\right)$ is the point of the genus $g=M-1$ hyperelliptic curve $\Gamma_{M-1}$, defined by (6.15). As it was mentioned in Section 5.5, all $u_{m}^{N}, w_{m}^{N}, u_{m}^{\prime N}$, and $w_{m}^{\prime N}$ may be parameterized in terms of $\theta$-functions on $\operatorname{Jac}\left(\Gamma_{M-1}\right)$.

The operator $\mathbf{Q}_{\phi}$ makes the isospectrality transformation (6.8) of the initial inhomogeneous quantum chain. So it may be decomposed similar to (2.41). If $\left|\Psi_{t}\right\rangle$ is the complete basis of the eigenvectors of $\mathbf{t}(\lambda)$, then

$$
\mathbf{Q}_{\phi}=\sum_{t}\left|\Psi_{t}\right\rangle q_{t, \phi}\left\langle\Psi_{t}^{\prime}\right|
$$


Using further the explicit formula (5.32) for the matrix elements of $\mathbf{Q}_{\phi}$ and the properties (4.5) of $w_{p}$-functions, we may calculate (taking the matrix elements)

$$
\mathbf{X Q}_{\phi} \mathbf{X}^{-1}=\mathbf{Q}_{\phi}\left[y_{1, m} \longmapsto \omega^{-1} y_{1, m}\right]
$$

And besides

$$
\begin{aligned}
\mathbf{Y Q}_{\phi}= & \mathbf{Q}_{\phi} \mathbf{Y} \\
= & \prod_{m \in \mathbb{Z}_{M}}\left(-\omega^{-1 / 2} \frac{y_{1, m}}{y_{3, m}} \frac{1-\omega x_{3, m}}{1-\omega x_{1, m}}\right) \\
& \cdot \mathbf{Q}_{\phi}\left[x_{1, m}, y_{1, m}, x_{3, m} \longmapsto \omega x_{1, m} \omega^{-1} y_{1, m}, \omega x_{3, m}\right] .
\end{aligned}
$$

The square braces in the right-hand sides of these two equations show the changes of the Fermat curve points. The rest $x_{j, m}$ and $y_{j, m}$ are the same in the left- and right-hand sides.

A glance to the parametrization (5.30) shows that the simultaneous change of the phases of all, say, $y_{1, m}$ is equivalent to the change of $\lambda_{\phi}$ while $\delta_{\phi}, \mu_{\phi}$, and $w_{\phi}$ remain unchanged. Using (6.18), we may conclude

$$
\mathbf{X Q}_{\phi} \mathbf{X}^{-1}=\sum_{t}\left|\Psi_{t}\right\rangle q_{t, \phi}\left(\lambda_{\phi} \longmapsto \omega^{-1} \lambda_{\phi}\right)\left\langle\Psi_{t}^{\prime}\right|
$$

Besides, both $\left|\Psi_{t}\right\rangle$ and $\left\langle\Psi_{t}^{\prime}\right|$ are the eigenvectors of $\mathbf{Y}, Y\left|\Psi_{t}\right\rangle=\left|\Psi_{t}\right\rangle \omega^{\gamma}$. The operator equation (6.13) may be rewritten as the functional equation

$$
t(\lambda) q_{t}(\lambda, \mu)=\mu^{-1} q_{t}\left(\omega^{-1} \lambda, \mu\right)+\frac{\mu}{\left(-\omega^{1 / 2} \lambda\right)^{N}} \omega^{\gamma} q_{t}(\omega \lambda, \mu),
$$

where $\left(\lambda^{N}, \mu^{N}\right) \in \Gamma_{M-1},(6.15)$, and in the decomposition $t(\lambda)=\sum_{m=0}^{M} \lambda^{-m} t_{m}, t_{M}=$ $(-\kappa)^{M} \omega^{\gamma}$.

Note that (6.21) does not follow from (6.13) directly. In general, coefficients $q_{t, \phi}=$ $\left\langle\Psi_{t}\left|\mathbf{Q}_{\phi}\right| \Psi_{t}^{\prime}\right\rangle$ in (6.13) may have the following structure:

$$
q_{t \phi}=q_{t}\left(\lambda_{\phi}, \mu_{\phi} ;\left\{u_{m}, w_{m}\right\}_{m=1}^{M},\left\{u_{m}^{\prime}, w_{m}^{\prime}\right\}_{m=1}^{M}\right) .
$$

Since the matrix elements of $\mathbf{Q}_{\phi}$ are the rational functions of $\lambda_{\phi}$ and of $\mu_{\phi}, q_{t}$ is a rational function of $\lambda_{\phi}$ and $\mu_{\phi}$. Therefore due to (6.20) the matrix elements of (6.13) between $\left\langle\Psi_{t}\right|$ and $\left|\Psi_{t}^{\prime}\right\rangle$ are the system of $N$ linear equations for $q_{t}\left(\omega^{k} \lambda_{\phi}, \mu_{\phi} ; \ldots\right), k \in$ $\mathbb{Z}_{N}$. Since $\left(\lambda_{\phi}^{N}, \mu_{\phi}^{N}\right) \in \Gamma_{M-1}$, the rank of this linear system is equal to $N-1$ for generic $\lambda_{\phi}$ (see $[27,28,29]$ for the details). Hence the linear system has a unique solution in the class of the rational functions of $\lambda_{\phi}, \mu_{\phi}$ up to a multiplier, depending on $\mu_{\phi}$ in general. We will show further that such multiplier may be fixed by an extra relation provided by (6.19).

Actually, there are two spectral curves in our considerations. The first one is the classical spectral curve $\Gamma_{M-1}$ with the point $\left(\lambda_{\phi}^{N}, \mu_{\phi}^{N}\right),(6.15)$. But the point $\left(\lambda_{\phi}, \mu_{\phi}\right)$ belongs to the quantum curve, which is $N^{2}$-sheeted covering of the classical one.

The considerations above prove the following proposition. 
Proposition 6.1. For any isospectral transformation $\left\{u_{m}, w_{m}\right\}_{m=1}^{M} \mapsto\left\{u_{m}^{\prime}, w_{m}^{\prime}\right\}_{m=1}^{M}$ the coefficients $q_{t}$ of the corresponding modified $Q$-operator, decomposed as in (6.17), depend only on the moduli of the spectral curve and on the point on the spectral curve

$$
q_{t, \phi}=q_{t}\left(\lambda_{\phi}, \mu_{\phi}\right)
$$

and do not depend on "classical times" (i.e., a point on the Jacobian), entering to a parameterization of $u_{m}, w_{m}, u_{m}^{\prime}, w_{m}^{\prime}$. In particular, for the homogeneous initial state, $q_{t}$ does not depend on the amplitudes of the solitonic states involved.

This proposition clarifies the derivation of (6.21) from (6.13).

6.2. Baxter equation for the homogeneous chain. All the formulas from the previous subsection are valid for the homogeneous initial state (2.23). Arbitrary value of $\lambda_{\phi}$ provides the trivial action of the functional counterpart $2_{\phi}$ of $\mathbf{Q}_{\phi}$, see discussion after Proposition 5.1. Such $\mathbf{Q}$-operators commute with $\mathbf{t}(\lambda)$ and form the commutative family. Explicit form of this $\mathbf{Q}$-operator is given by (5.32) with the homogeneous parameterization $p_{1, m}=p_{1}, p_{2, m}=p_{2}, p_{3, m}=p_{3}$,

$$
\begin{gathered}
x_{1}=\omega^{-1 / 2} \frac{\delta_{\phi}}{\kappa}, \quad x_{2}=\omega^{-1 / 2} \kappa, \quad x_{3}=\omega^{-1} \delta_{\phi}, \\
\frac{y_{3}}{y_{2}}=w_{\phi}, \quad \frac{y_{1}}{y_{3}}=\omega^{1 / 2} \frac{\lambda_{\phi}}{\kappa \delta_{\phi}},
\end{gathered}
$$

where $\delta_{\phi}, \lambda_{\phi}$, and $w_{\phi}$ are given by (2.30), (5.5), and (5.19) with generic value of $\phi$. Due to (6.24)

$$
\mathbf{Q}=\mathbf{Q}\left(\lambda_{\phi}, \delta_{\phi}\right)
$$

Several properties of this $\mathbf{Q}$-operator may be derived with the help of the matrix elements of (5.32) and (6.24), and with the help of (4.5). In particular, (6.18) and (6.19) become

$$
\begin{gathered}
\mathrm{XQ}\left(\lambda_{\phi}, \delta_{\phi}\right) \mathbf{X}^{-1}=\mathbf{Q}\left(\omega^{-1} \lambda_{\phi}, \delta_{\phi}\right) \\
\mathbf{Y Q}\left(\lambda_{\phi}, \delta_{\phi}\right)=\mathbf{Q}\left(\lambda_{\phi}, \delta_{\phi}\right) \mathbf{Y}=\left(-\frac{\lambda_{\phi}}{\delta_{\phi}} \frac{1-\delta_{\phi}}{\kappa-\omega^{1 / 2} \delta_{\phi}}\right)^{M} \mathbf{Q}\left(\lambda_{\phi}, \omega \delta_{\phi}\right) .
\end{gathered}
$$

The second equation in (6.26) is the consequence of (6.19) since

$$
x_{1}, y_{1}, x_{3} \longmapsto \omega x_{1}, \omega^{-1} y_{1}, \omega x_{3} \Longleftrightarrow \delta_{\phi} \longmapsto \omega \delta_{\phi} .
$$

Using (6.26) and (6.13), we obtain

$$
\mathbf{t}\left(\lambda_{\phi}\right) \mathbf{Q}\left(\lambda_{\phi}\right)=\delta_{\phi}^{-M} \mathbf{Q}\left(\omega^{-1} \lambda_{\phi}\right)+\left(-\omega^{-1 / 2} \frac{\delta_{\phi}}{\lambda_{\phi}}\right)^{M} \mathbf{Y Q}\left(\omega \lambda_{\phi}\right),
$$

where $\delta_{\phi}$-argument of all $\mathbf{Q}$ 's remains unchanged. Recall that $\mathbf{t}$ and $\mathbf{Q}$ may be diagonalized simultaneously. Comparing (6.28) and (6.13), we conclude $\mu_{\phi} \equiv \delta_{\phi}^{M}$. Further we will omit the subscribe $\phi$. Let $t(\lambda)$ and $q_{t}(\lambda, \delta)$ be the eigenvalues of $\mathbf{t}(\lambda)$ and $\mathbf{Q}(\lambda)$ for the same eigenvector, then (6.28) provides the functional equation

$$
t(\lambda) q_{t}(\lambda, \delta)=\delta^{-M} q_{t}\left(\omega^{-1} \lambda, \delta\right)+\left(-\omega^{-1 / 2} \frac{\delta}{\lambda}\right)^{M} \omega^{\gamma} q_{t}(\omega \lambda, \delta) .
$$


Here $q_{t}$ is a meromorphic function on the Baxter curve (see the set of definitions (2.31), (5.5), etc.)

$$
\left(\delta^{-1}, \delta^{*-1}\right) \in \Gamma_{B} \Longleftrightarrow \frac{1}{\delta^{N}}+\frac{1}{\delta^{* N}}=1-\kappa^{N} \frac{1}{\delta^{N} \delta^{* N}}, \quad \lambda=\delta \delta^{*} .
$$

More detailed investigation of (6.29) in the spirit of Proposition 6.1 shows that for generic $\lambda$ any solution of (6.29) such that $t(\lambda)$ is a polynomial and $q_{t}(\lambda, \delta)$ is a meromorphic function on the curve (6.30), gives the eigenvalue of $\mathbf{t}(\lambda)$ and $\mathbf{Q}(\lambda)$. The second equation of (6.26) provides the equation, fixing the $\delta$ ambiguity of the solution of the Baxter equation (6.29)

$$
\omega^{\gamma} q_{t}(\lambda, \delta)=\left(-\frac{\lambda}{\delta} \frac{1-\delta}{\kappa-\omega^{1 / 2} \delta}\right)^{M} q_{t}(\lambda, \omega \delta) .
$$

6.3. Evolution operator. $2_{\phi}$-transformation and its quantum counterpart $\mathbf{Q}_{\phi}$ may be interpreted as a kind of evolution, depending strongly on their spectral parameter $\lambda_{\phi}$. Evolution in more usual sense may be obtained in the limit when $\lambda_{\phi}^{-1} \mapsto 0$. Such a phenomenon, when a physical evolution operator is $Q$-operator in the singular point, appeared for example in the quantum Liouville model [8].

Evolution transformation $\mathbb{E}$ for the quantum relativistic Toda chain may be produced by nonlocal similarity transformation of the quantum $L$-operators (2.1)

$$
\mathfrak{E}\left(\ell_{m}\right)(\lambda)=\mathbf{A}_{m}(\lambda)^{-1} \ell_{m}(\lambda) \mathbf{A}_{m+1}(\lambda),
$$

where $2 \times 2$ matrix $\mathbf{A}_{m}$ has the operator-valued entries

$$
\mathbf{A}_{m}(\lambda)=\left(\begin{array}{cc}
1 & -\lambda^{-1} \mathbf{w}_{m-1}^{-1} \\
\mathbf{w}_{m} & -\omega^{-1 / 2} \kappa \lambda^{-1}
\end{array}\right) .
$$

The similarity transformations do not change the transfer matrix of the model with the periodical boundary conditions. The explicit form of $\mathfrak{E}$-transformation may be obtained from (6.32)

$$
\begin{gathered}
\mathfrak{E}\left(\mathbf{u}_{m}\right)=\omega^{-1 / 2} \mathbf{w}_{m}^{-1}, \\
\mathbb{E}\left(\mathbf{w}_{m}\right)=\left(1-\omega^{-1 / 2} \kappa \mathbf{w}_{m}^{-1} \mathbf{w}_{m-1}\right)^{-1} \omega^{1 / 2} \mathbf{w}_{m-1} \mathbf{u}_{m} \mathbf{w}_{m+1}\left(1-\omega^{-1 / 2} \kappa \mathbf{w}_{m+1}^{-1} \mathbf{w}_{m}\right) .
\end{gathered}
$$

We may check directly that $\mathbb{E}$-transformation is the canonical one. As usual, at the root of unity we have to separate

$$
\mathfrak{E}(f)=\mathbf{E} \mathscr{\mathscr { C }}(f) \mathbf{E}^{-1},
$$

where $\mathbf{E}$ is the finite-dimensional operator, and $\mathscr{E}$ acts on $N$ th powers of the Weyl elements $\mathbf{u}_{m}^{N}, \mathbf{w}_{m}^{N}$ as follows:

$$
\begin{gathered}
\mathscr{E}\left(u_{m}^{N}\right)=-\frac{1}{w_{m}^{N}} \\
\mathscr{E}\left(w_{m}^{N}\right)=-u_{m}^{N} w_{m}^{N} w_{m-1}^{N} \frac{w_{m+1}^{N}+\kappa^{N} w_{m}^{N}}{w_{m}^{N}+\kappa^{N} w_{m-1}^{N}}
\end{gathered}
$$


For the given set of $\left\{u_{m}, w_{m}\right\}_{m=1}^{M}$ parameterized in terms of $\tau_{m}$ and $\theta_{m},(5.15)$, denote

$$
\tau_{m}=\tau_{0, m}, \quad \theta_{m}=\tau_{1, m},
$$

where we have introduced the extra auxiliary subscript of $\tau$-function. The first equation in (6.36) yields

$$
\mathscr{E}\left(\boldsymbol{\tau}_{l, m}\right)=\boldsymbol{\tau}_{l+1, m},
$$

and the second equation in (6.36) gives the following "equation of motion":

$$
\boldsymbol{\tau}_{l, m+1}^{N} \boldsymbol{\tau}_{l, m-1}^{N}+\kappa^{N}\left(\boldsymbol{\tau}_{l, m}^{N}\right)^{2}=\left(1+\kappa^{N}\right) \boldsymbol{\tau}_{l+1, m}^{N} \boldsymbol{\tau}_{l-1, m}^{N} .
$$

Let $\tau_{0, m}^{N}=\tau_{m}^{N}$ given by the $n$-solitonic expression (5.21). Then the complete solution of (6.39) is given by

$$
\left(\tau_{l, m}^{(n)}\right)^{N}=h^{(n)}\left(\left\{f_{k} \mathbf{e}^{2 i m \phi_{k}+2 i l \psi_{k}}\right\}_{k=1}^{n}\right),
$$

where

$$
\mathbf{e}^{2 i \psi_{k}}=s_{k}(1)=\frac{\Delta_{k}^{*}}{\Delta_{k}} \frac{\Delta_{k}-1}{\Delta_{k}^{*}-1},
$$

see (5.23) and (5.24) for the notations. The substitution (6.40) makes (6.39) the identity (see the appendix).

Comparing (6.40) with the definition of $h^{(g)}$ and $\tau_{m}^{(g)}$, (5.21), (5.23), we see that in fact

$$
\tau_{l, m}^{(n)}=\tau_{m}^{(n+l)},
$$

where the initial set of $\left\{\phi_{k}, f_{k}\right\}_{k=1}^{n}$ for $h^{(n)}$ and $\tau_{m}^{(n)}$ is enlarged formally by $\left\{\phi_{k}^{\prime}, f_{k}^{\prime}\right\}_{k=n+1}^{n+l}$ with $f_{k}^{\prime} \equiv 0$ and all $\phi_{k}^{\prime}$ equal and obeying formally

$$
\delta_{\phi_{k}^{\prime}}^{* N}=1 \Longrightarrow \delta_{\phi_{k}^{\prime}}^{-1}=\lambda_{\phi_{k}^{\prime}}^{-1}=0 .
$$

This establishes the relation between the evolution (6.34), (6.36) and $2_{\phi}, \mathbf{Q}_{\phi}$. In particular, the finite-dimensional counterpart $\mathbf{E}$ of the evolution operator $\mathbb{E}$ may be obtained in the limit

$$
\mathbf{E}=\lim _{\delta=\infty, \delta^{*}=1} \mathbf{Q}\left(\delta, \delta^{*}\right) .
$$

Equation (5.32) gives the explicit form of the matrix elements of $\mathbf{E}$ on the $l$ th step of the evolution

$$
\left\langle\alpha\left|\mathbf{E}_{l}\right| \beta\right\rangle=\prod_{m=1}^{M} \omega^{\left(\alpha_{m}-\beta_{m+1}\right) \beta_{m}} w_{p_{2, l, m}}\left(\beta_{m+1}-\beta_{m}\right),
$$

where

$$
x_{2, l, m}=\omega^{-1 / 2} \kappa \frac{\boldsymbol{\tau}_{l, m}^{2}}{\boldsymbol{\tau}_{l, m-1} \boldsymbol{\tau}_{l, m+1}}, \quad y_{2, l, m}=\kappa^{\prime} \frac{\boldsymbol{\tau}_{l-1, m} \boldsymbol{\tau}_{l+1, m}}{\boldsymbol{\tau}_{l, m-1} \boldsymbol{\tau}_{l, m+1}}
$$


with

$$
\kappa^{\prime N}=1+\kappa^{N}
$$

In particular, $N$-bilinear relation (6.39) allows to parameterize the Fermat relation for the coordinates of $p_{j, m}$. Let

$$
\bar{\theta}_{m}=\tau_{-1, m}
$$

Then

$$
\begin{gathered}
y_{1, m}^{(n)}=\omega^{1 / 2} \frac{\lambda_{n} w_{\phi_{n}} \kappa^{\prime}}{\kappa \delta_{n}} \frac{\bar{\theta}_{m}^{(n)} \theta_{m-1}^{(n-1)}}{\tau_{m}^{(n)} \tau_{m-1}^{(n-1)}} \\
y_{2, m}^{(n)}=\kappa^{\prime} \frac{\bar{\theta}_{m}^{(n)} \theta_{m}^{(n)}}{\tau_{m-1}^{(n)} \tau_{m+1}^{(n)}}, \quad y_{3, m}^{(n)}=w_{\phi_{n}} \kappa^{\prime} \frac{\bar{\theta}_{m}^{(n)} \theta_{m}^{(n-1)}}{\tau_{m+1}^{(n)} \tau_{m-1}^{(n-1)}}
\end{gathered}
$$

7. Quantum separation of variables. Turn to the last subject of this paper-the quantum separation of variables, or functional Bethe ansatz.

In the previous sections we have established the following: let $\mathbf{t}(\lambda) \equiv \mathbf{t}^{(0)}(\lambda)$ be the transfer matrix of the homogeneous quantum chain of the length $M$. There exists the family of isospectral to $\mathbf{t}(\lambda)$ transfer matrices $\mathbf{t}^{(M-1)}(\lambda)$, parameterized by $(M-1)$ complex variables $f_{1}, \ldots, f_{M-1}$-the amplitudes of Hirota-type solitons in the minimal set (5.29). The isospectrality means that for any generic values of $f_{1}, \ldots, f_{M-1} \mathbf{t}^{(M-1)}(\lambda)$ has the same eigenvalues as the initial $\mathbf{t}(\lambda)$. Therefore only the eigenvectors $\left|\Psi_{t}^{(M-1)}\right\rangle$ depend on $f_{1}, \ldots, f_{M-1}$. We may choose the values of $f_{1}, \ldots, f_{M-1}$ in an appropriate way, such that $\left|\Psi_{t}^{(M-1)}\right\rangle$ would become more simple than the initial unknown $\left|\Psi_{t}\right\rangle$. Since the similarity matrix $\mathbf{K}^{(M-1)}$ is known explicitly, any exact information about $\left|\Psi^{(M-1)}\right\rangle$ solves the eigenstate problem for the initial homogeneous quantum relativistic Toda chain.

7.1. Null subspaces of $\mathbf{b}(\lambda)$. Now we have to understand what special values of $f_{1}, \ldots, f_{M-1}$ are the most useful ones. To do this, we should turn back to the degenerated $\tilde{\ell}_{\phi}$-operator and write out (6.3) for the monodromies of $\ell$ and $\mathbf{R}$

$$
\psi_{\phi, 1}^{\prime *} \cdot \hat{\mathbf{t}}\left(\lambda_{\phi}\right) \hat{\mathbf{Q}}_{\phi}=\hat{\mathbf{Q}}_{\phi}^{\prime} \psi_{\phi, M+1}^{\prime *}, \quad \hat{\mathbf{t}}\left(\lambda_{\phi}\right) \hat{\mathbf{Q}}_{\phi} \cdot \psi_{\phi, M+1}^{\prime \prime}=\psi_{\phi, 1}^{\prime \prime} \hat{\mathbf{Q}}_{\phi}^{\prime \prime} .
$$

In components,

$$
\begin{gathered}
\left(\mathbf{a}\left(\lambda_{\phi}\right)-\omega^{1 / 2} \frac{u_{\phi, 1}}{\lambda_{\phi}} \mathbf{x}_{\phi} \mathbf{c}\left(\lambda_{\phi}\right)\right) \hat{\mathbf{Q}}_{\phi}=\hat{\mathbf{Q}}_{\phi}^{\prime}, \\
\left(\mathbf{b}\left(\lambda_{\phi}\right)-\omega^{1 / 2} \frac{u_{\phi, 1}}{\lambda_{\phi}} \mathbf{x}_{\phi} \mathbf{d}\left(\lambda_{\phi}\right)\right) \hat{\mathbf{Q}}_{\phi}=-\omega^{1 / 2} \frac{u_{\phi, M+1}}{\lambda_{\phi}} \hat{\mathbf{Q}}_{\phi}^{\prime} \mathbf{x}_{\phi}, \\
\mathbf{a}\left(\lambda_{\phi}\right) \hat{\mathbf{Q}}_{\phi} \omega^{1 / 2} \frac{u_{\phi, M+1}}{\lambda_{\phi}} \mathbf{x}_{\phi}+\mathbf{b}\left(\lambda_{\phi}\right) \hat{\mathbf{Q}}_{\phi}=\omega^{1 / 2} \frac{u_{\phi, 1}}{\lambda_{\phi}} \mathbf{x}_{\phi} \hat{\mathbf{Q}}_{\phi}^{\prime \prime}, \\
\mathbf{c}\left(\lambda_{\phi}\right) \hat{\mathbf{Q}}_{\phi} \omega^{1 / 2} \frac{u_{\phi, M+1}}{\lambda_{\phi}} \mathbf{x}_{\phi}+\mathbf{d}\left(\lambda_{\phi}\right) \hat{\mathbf{Q}}_{\phi}=\hat{\mathbf{Q}}_{\phi}^{\prime \prime} .
\end{gathered}
$$


Using the relation $\mathbf{R}_{m, \phi} \mathbf{x}_{m}=\mathbf{x}_{\phi} \mathbf{R}_{m, \phi}$ (see (4.11)) we may obtain from (6.4)

$$
\begin{aligned}
& \hat{\mathbf{Q}}_{\phi}^{\prime}=\left(\prod_{m=1}^{M} \frac{u_{m}}{u_{\phi, m+1}}\right) \mathbf{X} \hat{\mathbf{Q}}_{\phi} \mathbf{X}^{-1} \mathbf{x}_{1} \mathbf{x}_{\phi}^{-1}, \\
& \hat{\mathbf{Q}}_{\phi}^{\prime \prime}=\left(\prod_{m=1}^{M} \omega^{1 / 2} \frac{u_{\phi, m+1} w_{m}}{\lambda_{\phi}}\right) \mathbf{Z} \hat{\mathbf{Q}}_{\phi} \mathbf{X} \mathbf{x}_{1}^{-1} \mathbf{x}_{\phi} .
\end{aligned}
$$

Let the initial chain is the homogeneous one. If $\phi \in \mathfrak{f}_{M}$, then

$$
u_{\phi, m}^{N}=u_{\phi, M+m}^{N}=(-)^{N-1} \delta_{\phi}^{N} \frac{1-f_{\phi} \mathbf{e}^{2 i \phi(m-1)}}{1-f_{\phi} \mathbf{e}^{2 i \phi m}} .
$$

Note that

$$
\prod_{m \in Z_{M}} u_{\phi, m}=\left(-\omega^{-1 / 2} \delta_{\phi}\right)^{M}
$$

does not depend on $f_{\phi}$. Consider the limit when $f_{\phi} \mapsto 1$. Due to (7.5) $\hat{\mathbf{Q}}_{\phi}, \hat{\mathbf{Q}}_{\phi}^{\prime}$, and $\hat{\mathbf{Q}}_{\phi}^{\prime \prime}$ are regular, but

$$
u_{\phi, 1}=u_{\phi, M+1}=0
$$

and (7.2) give

$$
\mathbf{a}\left(\lambda_{\phi}\right) \tilde{\mathbf{Q}}_{\phi}=\tilde{\mathbf{Q}}_{\phi}^{\prime}, \quad \mathbf{d}\left(\lambda_{\phi}\right) \tilde{\mathbf{Q}}_{\phi}=\tilde{\mathbf{Q}}_{\phi}^{\prime \prime}, \quad \mathbf{b}\left(\lambda_{\phi}\right) \widetilde{\mathbf{Q}}_{\phi}=0 .
$$

The last expression is important. Taking the trace over $\phi$ th space, we obtain

$$
\mathbf{b}\left(\lambda_{\phi}\right) \mathbf{Q}_{\phi}=0 \text { if } f_{\phi}=1 .
$$

Recall that the phase of $\lambda_{\phi}$ is the parameters of $\mathbf{Q}_{\phi}$.

Turn now to the operator $\mathbf{K}^{(M-1)}$ and consider the limit when all $f_{n} \mapsto 1, n=$ $1, \ldots, M-1$. Each $\mathbf{Q}_{\phi_{n}}$ in (3.25) depends essentially on the phase of $\lambda_{\phi_{n}}, n=1, \ldots, M-1$. Since $\mathbf{K}^{(M-1)}$ is the symmetrical operator with respect to any permutation of $\left\{\left(f_{n}, \phi_{n}\right)\right\}_{n=1}^{M-1}$, see Proposition 5.2, the following proposition holds.

Proposition 7.1. Let $\lambda_{\phi_{n}}$ be the spectral parameter of $\mathbf{Q}_{\phi_{n}}$ in the decomposition (3.25) of the minimal $\mathbf{K}^{(M-1)}$ (5.29). Then

$$
\mathbf{b}\left(\lambda_{\phi_{n}}\right) \mathbf{K}^{(M-1)}=0, \quad n=1, \ldots, M-1 \text { if all } f_{n} \longmapsto 1 .
$$

The structure of eigenvectors of $\mathbf{b}(\lambda)$ was described by (2.46). Comparing (2.46) and (7.9), and taking into account $\mathbf{Y} \mathbf{Q}_{\phi}=\mathbf{Q}_{\phi} \mathbf{Y}$ for any $\mathbf{Q}_{\phi}$, we may conclude that in the limit $f_{k} \mapsto 1$ for all $k$

$$
\mathbf{K}^{(M-1)}\left(\left\{\lambda_{\phi_{k}}\right\}_{k=1}^{M-1}\right)=\sum_{\gamma \in \mathbb{Z}_{N}}\left|\left\{\lambda_{k}\right\}_{k=1}^{M-1}, \gamma\right\rangle\left\langle\chi_{\gamma}\right| .
$$

Here $\left|\left\{\lambda_{k}\right\}_{k=1}^{M-1}, \gamma\right\rangle$ are the eigenvectors of $\tilde{\mathbf{b}}$ (2.45), (2.46), and $\chi_{\gamma}$ are some vectors, such that $\left\langle\chi_{\gamma}\right| \mathbf{Y}=\omega^{\gamma}\left\langle\chi_{\gamma}\right|$, where $\mathbf{Y}$ is given by (2.15). 
From the other side, using the natural decomposition (3.21) and (2.41), we should have for the generic values of $f_{k}$

$$
\mathbf{K}^{(M-1)}\left(\left\{\lambda_{\phi_{k}}\right\}_{k=1}^{M-1}\right)=\sum_{t}\left|\Psi_{t}\right\rangle\left(\prod_{k=1}^{M-1} q_{t}\left(\lambda_{\phi_{n}}, \delta_{\phi_{n}}\right)\right)\left\langle\Psi_{t}^{(M-1)}\right|,
$$

where the summation is taken over all the eigenvalues $t$ of $\mathbf{t}$. Functions $q_{t}(\lambda, \delta)$ obey the Baxter equation (6.29) and due to Proposition 6.1 do not depend on $f_{1}, \ldots, f_{M-1}$. Comparing the two decompositions (7.10) and (7.11) at $f_{k} \mapsto 1$, we may conclude that the eigenvectors $\left\langle\Psi_{t}^{(M-1)}\right|$ stick together in this limit

$$
\left\langle\Psi_{t}^{(M-1)}\right| \longmapsto\left\langle\chi_{\gamma}\right| \quad \forall t=\left\{t_{1}, \ldots, t_{M-1}, t_{M}=(-\kappa)^{M} \omega^{\gamma}\right\} .
$$

So the functional Bethe ansatz formula (2.47) follows from (7.10) and (7.11) up to a normalization. In addition, we know explicitly the corresponding projector $\mathbf{K}^{(M-1)}$.

Detailed investigation shows that the explicit form of $\left\langle\chi_{y}\right|$ depends on a limiting procedure $f_{n} \mapsto 1$. The reason is that when $f_{n} \mapsto 1, n=1, \ldots, g$, then $\tau_{m}^{(g)} \mapsto 0, m=$ $0, \ldots, g-1$. This follows from the recursive definition of $h^{(g)}(5.23)$. The proof is based on Proposition .1 in the appendix. Because of the parameterization of $u_{m}^{(n)}$ and $p_{j, m}^{(n)}$ contains the ratios of $\tau_{m}^{(n)}$, the ambiguity arises. Some of $u_{m}^{(n)}$ and $p_{j, m}^{(n)}$ of the form $0 / 0$ depends on the details of the limiting procedure.

7.2. Limiting procedure. In this subsection, we describe one of the possible limiting procedures. Most simple $\mathbf{K}^{(M-1)}\left(\left\{\lambda_{k}\right\}\right)$ appears when we choose $u_{m}^{(M-1)}=0$ for $m=1, \ldots, M-1$. Consider the set of infinitely small numbers,

$$
\varepsilon_{k} \longmapsto 0, \quad k=1, \ldots, M-1,
$$

such that any ratio $\varepsilon_{n} / \varepsilon_{m} \neq 1$ is finite, and

$$
\begin{aligned}
& u_{m}^{(M-1)}=-\omega^{-1 / 2} \frac{\varepsilon_{M-m}}{\delta_{1} \cdots \delta_{M-1}}, \quad m=1, \ldots, M-1, \\
& u_{M}^{(M-1)}=-\omega^{-1 / 2} \frac{\left(\delta_{1} \cdots \delta_{M-1}\right)^{M-1}}{\varepsilon_{1} \cdots \varepsilon_{M-1}} .
\end{aligned}
$$

The recursion (3.11), (3.12) with

$$
\kappa_{\phi_{n}}=\frac{\kappa}{\delta_{n}}
$$

yields

$$
u_{m}^{(n)}=-\omega^{-1 / 2} \frac{\varepsilon_{n-m+1}}{\delta_{1} \cdots \delta_{n}}+o\left(\varepsilon_{1}\right), \quad m=1 \cdots n
$$

while for $m=n+1, \ldots, M-1$ all $u_{m}^{(n)}$ are regular. This gives

$$
\boldsymbol{\tau}_{m}^{(n)}=\boldsymbol{\tau}_{n}^{(n)} \frac{\varepsilon_{1} \cdots \varepsilon_{n-m}}{\left(\delta_{1} \cdots \delta_{n}\right)^{n-m}}+o\left(\varepsilon_{1}^{n-m}\right), \quad m=0, \ldots, n .
$$


In the limit $\varepsilon_{k}=0$ we may choose

$$
\frac{\tau_{n}^{(n)}}{\theta_{n}^{(n)}}=\prod_{k=1}^{n} \omega^{-1 / 2} \frac{\delta_{k}}{\lambda_{k} w_{k}},
$$

where $w_{k}$ are given by (5.19), and

$$
\theta_{m}^{(n)}=\left(\omega^{1 / 2} \kappa\right)^{(n-m)(n-m-1) / 2} \frac{\theta_{n}^{(n)}}{\left(\delta_{1} \cdots \delta_{n}\right)^{n-m}} .
$$

The Nth powers of these formulas follow from Proposition .3. Equation (7.19) gives

$$
\frac{w_{m+1}^{(n)}}{w_{m}^{(n)}}=\omega^{1 / 2} \kappa, \quad m=0, \ldots, n-1 .
$$

In the limit when $\epsilon_{k} \mapsto 0$ the regular $p_{j, m}^{(n)}, j=1,2,3$, in $\mathbf{Q}_{\phi_{n}}$ (5.32), entering the decomposition of $\mathbf{K}^{(M-1)}$, are given by (5.30). Below we will list the singular $p_{j, m}^{(n)}$, using notations (4.6) and (4.7):

(i) $m=1 \cdots n-1$,

$$
p_{3, m}^{(n)}=q_{1}, \quad p_{2, m}^{(n)}=O\left(p_{1, m}^{(n)}\right), \quad x_{1, m}^{(n)}=\frac{1}{\omega^{1 / 2} \kappa} \frac{\varepsilon_{n-m+1}}{\varepsilon_{n-m}} .
$$

(ii) $m=n$,

$$
p_{1, n}^{(n)}=q_{0}, \quad p_{2, n}^{(n)}=q_{\infty} \quad \forall n,
$$

and $p_{3, n}^{(n)}$ are regular, except

$$
p_{3, M-1}^{(M-1)}=q_{\infty} .
$$

(iii) $m=n+1 \cdots M-2$ : all $p_{j, m}^{(n)}$ in this region are regular.

(iv) $m=M-1: p_{1, M-1}^{(n)}$ are regular except

$$
p_{1, M-1}^{(M-1)}=q_{0}, \quad p_{2, M-1}^{(n)}=p_{3, M-1}^{(n)}=q_{\infty} \quad \forall n .
$$

(v) $m=M$,

$$
p_{1, M}^{(n)}=q_{\infty}, \quad p_{2, M}^{(n)}=p_{3, M}^{(n)}=q_{0} \quad \forall n .
$$

Substituting these expressions into (5.32), we obtain the following form of $N$ th modified $Q$-matrix, $n \neq M-1$ :

$$
\begin{aligned}
\left\langle\alpha\left|\mathbf{Q}^{(n)}\right| \beta\right\rangle= & \frac{1}{\Phi\left(\beta_{1}+\beta_{M}-\alpha_{M}\right)} \prod_{m=1}^{n-1} \delta_{\alpha_{m}, \beta_{m+1}} \Phi\left(\alpha_{n}\right) w_{O p_{3, n}^{(n)}}\left(\alpha_{n}-\beta_{n+1}\right) \\
& \times \prod_{m=n+1}^{M-2} \omega^{\left(\alpha_{m}-\beta_{m}\right) \beta_{m+1}} \frac{w_{p_{1, m}^{(n)}}\left(\beta_{m}-\alpha_{m}\right) w_{p_{2, m}^{(n)}}\left(\beta_{m+1}-\beta_{m}\right)}{w_{p_{3, m}^{(n)}}\left(\beta_{m+1}-\alpha_{m}\right)} \\
& \times \frac{\Phi\left(\alpha_{M-1}\right)}{\Phi\left(\beta_{M-1}\right)} w_{p_{1, M-1}^{(n)}}\left(\beta_{M-1}-\alpha_{M-1}\right)
\end{aligned}
$$


and for the last $(M-1)$ th $Q$-matrix

$$
\left\langle\alpha\left|\mathbf{Q}^{(M-1)}\right| \beta\right\rangle=\frac{1}{\Phi\left(\beta_{1}+\beta_{M}-\alpha_{M}\right)} \prod_{m=1}^{M-2} \delta_{\alpha_{m}, \beta_{m+1}} \Phi\left(\alpha_{M-1}\right) .
$$

Here $\Phi$ is given by (4.4). Explicit form of the modified $Q$-operators (7.26) and (7.27) allows to prove the following proposition.

Proposition 7.2. The matrix $\mathbf{K}^{(M-1)}$ obeys

$$
\mathbf{K}^{(M-1)}\left(\left\{\lambda_{k}\right\}_{k=1}^{M-1}\right)\left(\mathbf{z}_{m}-\mathbf{z}_{m+1}\right)=0, \quad m \in \mathbb{Z}_{M}
$$

Proof. The matrix elements of $\mathbf{Q}^{(n)}$ depend on $\beta_{M}-\alpha_{M}$, therefore $\mathbf{Q}^{(n)} \mathbf{Z}_{M}=$ $\mathbf{z}_{M} \mathbf{Q}^{(n)}$ for all $n$. The set of delta symbols in each $\mathbf{Q}^{(n)}$ gives $\mathbf{Q}^{(n)} \mathbf{z}_{m}=\mathbf{z}_{m-1} \mathbf{Q}^{(n)}$, $m=2, \ldots, n+1$. Also the matrix elements of each $\mathbf{Q}^{(n)}$ depend on $\beta_{1}+\beta_{M}$, hence $\mathbf{Q}^{(n)}\left(\mathbf{z}_{1}-\mathbf{z}_{M}\right)=0$ for all $n$. Therefore for all $m$

$$
\mathbf{K}^{(M-1)}\left(\mathbf{z}_{m}-\mathbf{z}_{M}\right)=\mathbf{Q}^{(1)} \cdots \mathbf{Q}^{(M-m)}\left(\mathbf{z}_{1}-\mathbf{z}_{M}\right) \mathbf{Q}^{(M-m+1)} \cdots \mathbf{Q}^{(M-1)}=0 .
$$

So, in our limiting procedure, $x_{\gamma}$ is defined by

$$
\left\langle\chi_{\gamma}\right|\left(\mathbf{z}_{m}-\mathbf{z}_{m+1}\right)=0, \quad\left\langle\chi_{\gamma}\right| \mathbf{Y}=\omega^{\gamma}\left\langle\chi_{\gamma}\right| .
$$

Hence the matrix element of $\chi_{\gamma}$ is

$$
\left\langle\chi_{\gamma} \mid \alpha\right\rangle=\chi_{\gamma}(\bar{\alpha}) \equiv \chi_{\gamma}(\bar{\alpha} \bmod N),
$$

where

$$
\bar{\alpha} \stackrel{\text { def }}{=} \sum_{m \in \mathbb{Z}_{M}} \alpha_{m} .
$$

The discrete function $\chi_{\gamma}(\bar{\alpha})$ is defined by

$$
\frac{\chi_{\gamma}(\bar{\alpha}+M)}{\chi_{\gamma}(\bar{\alpha})}=\left(-\omega^{-1 / 2}\right)^{M} \omega^{\gamma-\bar{\alpha}} .
$$

Solution of (7.33) depends strongly on $M \bmod N$.

8. Discussion. In this paper, we investigated one of the simplest integrable model, associated with the local Weyl algebra at the root of unity. All such models always contain a classical discrete dynamic of parameters. Nontrivial solution of this classical part of the model provides the solution of the isospectrality problem of the spin part. Well-known nowadays results by Sklyanin, Kuznetsov, and others, reveal that in the classical limit of the usual Toda chain (and many other models) Baxter's quantum $Q$-operator is related to the Bäcklund transformation of the classical system, see, for example, $[12,13,26,27,28,29,30]$ and so forth. In our case we have the Bäcklund transformation of the classical counterpart and modified $Q$-operator in the quantum space simultaneously. It is unusual that solving the quantum isospectrality problem, we miss the commutativity of the modified $Q$-operators. 
Our results are the explicit construction of $(M-1)$-parametric family of quantum inhomogeneous transfer matrices with the same spectrum as the initial homogeneous one and the explicit construction of the corresponding similarity operator (3.25). We hope that the solution of the isospectrality problem will help to solve the model with arbitrary $N$ in the thermodynamic limit exactly.

As one particular application of the isospectrality we have obtained the quantum separation of variables. Previously, there was a hypothesis formulated for the usual quantum Toda chain, that the product of operators $Q$, taken in the special points, is related to the functional Bethe ansatz. In this paper, we have established it explicitly, but for the product of modified operators $Q$.

Note in conclusion that this method may be applied to any model, based on the local Weyl algebra. We may mention the chiral Potts model [1, 4] and the ZamolodchikovBazhanov-Baxter model in the vertex formulation [25]. Moreover, all two-dimensional integrable models with the local Weyl algebra are particular cases of the general three dimensional model, and their classical counterparts are known [20, 21, 22, 23, 24].

\section{APPENDIX}

Fay identity in the rational limit. It is well known what is the rational limit of $\Theta$-function on a generic algebraic curve. $\Theta$-function by definition is

$$
\Theta^{(g)}(\mathbf{z})=\sum_{\mathbf{n} \in \mathbb{Z}^{g}} \mathbf{e}^{i \pi(\mathbf{n}, \Omega \mathbf{n})+2 i \pi(\mathbf{n}, \mathbf{z})},
$$

where $\mathbf{z}=\left(z_{1}, \ldots, z_{g}\right) \in \mathbb{C}^{g}$ and the imaginary part of the symmetrical period matrix $\Omega$ is positively defined. $\Theta$-function may be defined recursively as

$$
\Theta^{(g)}(\mathbf{z})=\sum_{n_{g} \in \mathbb{Z}} \Theta^{(g-1)}\left(\mathbf{z}^{\prime}+\Omega_{g}^{\prime} n_{g}\right) \mathbf{e}^{i \pi \Omega_{g, g} n_{g}^{2}+2 i \pi n_{h} z_{g}},
$$

where $\mathbf{z}^{\prime}=\left(z_{1}, \ldots, z_{g-1}\right)$ and $\Omega_{g}^{\prime}=\left(\Omega_{1, g}, \ldots, \Omega_{g-1, g}\right)$.

The rational limit corresponds to

$$
\begin{gathered}
\mathbf{e}^{i \pi \Omega_{n, n}+2 i \pi z_{n}}=-f_{n}, \\
\mathbf{e}^{i \pi \Omega_{n, n}} \longmapsto 0, \quad \mathbf{e}^{i \pi \Omega_{k, n}} \longmapsto \frac{\left(q_{k}-q_{n}\right)\left(p_{k}-p_{n}\right)}{\left(q_{k}-p_{n}\right)\left(p_{k}-q_{n}\right)} .
\end{gathered}
$$

Explicit details of the limiting procedure are rather tedious. We should better invent all the necessary components in the rational limit directly and formulate the rational limit of the Fay identity. The proof of all the propositions is analogous to that in the algebraic geometry with the elementary replacement of the usual $\Theta$-function methods to the analysis of the polynomial structure and intensive use of the main algebraic theorem.

.1. Rational limit of arbitrary algebraic curve Jacobian. Let $\left\{p_{n}, q_{n}\right\}_{n=1}^{g}$ be a set of $2 g$ complex parameters. " $\Theta$-function" in the rational limit is a function of $g$ free complex arguments $f_{1}, \ldots, f_{g}$, the set $\left\{p_{n}, q_{n}\right\}_{n=1}^{g}$ is its parameters. $\Theta$-function may 
be defined recursively with respect to $g$ as follows:

$$
\begin{gathered}
\Theta^{(0)}=1, \quad \Theta^{(1)}\left(f_{1}\right)=1-f_{1}, \\
\Theta^{(2)}\left(f_{1}, f_{2}\right)=1-f_{1}-f_{2}+d_{1,2} f_{1} f_{2},
\end{gathered}
$$

and so on,

$$
\Theta^{(g)}\left(\left\{f_{n}\right\}_{n=1}^{g}\right)=\Theta^{(g-1)}\left(\left\{f_{n}\right\}_{n=1}^{g-1}\right)-f_{g} \Theta^{(g-1)}\left(\left\{f_{n} d_{n, g}\right\}_{n=1}^{g-1}\right) .
$$

The recursion follows from (A.2), where $n_{g}=0,1$. Parameters $\left\{p_{n}, q_{n}\right\}_{n=1}^{g}$ enter the definition of the phase shifts

$$
d_{n, m}=\frac{\left(p_{n}-p_{m}\right)\left(q_{n}-q_{m}\right)}{\left(p_{n}-q_{m}\right)\left(q_{n}-p_{m}\right)} .
$$

It is useful to introduce the function $\sigma_{n}(z)$,

$$
\sigma_{n}(z)=\frac{p_{n}-z}{q_{n}-z}
$$

Let by definition

$$
H^{(g)}\left(\left\{f_{n}\right\}_{n=1}^{g}\right)=\Theta^{(g)}\left(\left\{f_{n} \prod_{m \neq n} \sigma_{n}\left(q_{m}\right)\right\}_{n=1}^{g}\right) .
$$

Recursion definition of $H^{(g)}$ is

$$
H^{(g)}\left(\left\{f_{n}\right\}_{n=1}^{g}\right)=H^{(g-1)}\left(\left\{f_{n} \sigma_{n}\left(q_{g}\right)\right\}_{n=1}^{g-1}\right)-f_{g} \prod_{m=1}^{g-1} \sigma_{g}\left(q_{m}\right) H^{(g-1)}\left(\left\{f_{n} \sigma_{n}\left(p_{g}\right)\right\}_{n=1}^{g-1}\right),
$$

where $\sigma_{n}\left(q_{g}\right) d_{n, g}=\sigma_{n}\left(p_{g}\right)$ is taken into account.

Proposition .1 (zeros of $\Theta$-function). The variety $H^{(g)}=0$ may be parametrized as follows:

$$
H^{(g)}\left(\left\{f_{n}\right\}_{n=1}^{g}\right)=0 \Longleftrightarrow f_{n}=\prod_{m=1}^{g-1} \sigma_{n}\left(z_{m}\right)^{-1},
$$

where $z_{m} \in \mathbb{C}, m=1, \ldots, g-1$.

Proof. We will use the mathematical induction method. This proposition is obviously valid for $g=1$. Suppose that we have $H^{(g-1)}\left(\left\{\prod_{m=1}^{g-2} \sigma_{n}\left(z_{m}\right)^{-1}\right\}_{n=1}^{g-1}\right)=0$ for all $z_{1} \ldots z_{m}, p_{1} \ldots p_{g-1}, q_{1} \ldots q_{g-1}$ for some $g$. Consider now $H^{(g)}$. Due to recursion relation (A.9)

$$
H^{(g)}\left(\left\{\prod_{m=1}^{g-1} \sigma_{n}\left(z_{m}\right)^{-1}\right\}_{n=1}^{g}\right)=\frac{P_{g}\left(z_{1}, \ldots, z_{m}\right)}{\prod_{n<n^{\prime}}\left(q_{n}-q_{n^{\prime}}\right) \prod_{n=1}^{g} \prod_{m=1}^{g-1}\left(p_{n}-z_{m}\right)},
$$

where $P_{\mathfrak{g}}$ is a polynomial of the power $g$ with respect to each $z_{m}$. 
Consider the case when $z_{g-1}=q_{g}$, so $\prod_{m} \sigma_{g}\left(z_{m}\right)^{-1}=0$. From recursion relation (A.9) and the supposition of the induction, it follows that $H^{(g)}=0$, so due to the symmetry of $H^{(g)}$ we obtain

$$
H^{(g)}\left(\left\{\prod_{m=1}^{g-1} \sigma_{n}\left(z_{m}\right)^{-1}\right\}_{n=1}^{g-1}\right)=0 \quad \text { if } z_{m}=q_{n} \forall n, m .
$$

Next consider the case when $z_{g-1} \mapsto p_{g}$, hence $\prod \sigma_{n}\left(z_{m}\right) \sim\left(p_{g}-z_{g-1}\right)^{-1} \mapsto \infty$. Due to recursion relation (A.9) and the supposition of the induction it follows that $H^{(g)}$ is regular in $z_{g-1}=p_{g}$. Again, due to the symmetry of $H^{(g)}$

$$
\operatorname{Res}_{z_{m}=p_{n}} H^{(g)}\left(\left\{\prod_{m=1}^{g-1} \sigma_{n}\left(z_{m}\right)^{-1}\right\}_{n=1}^{g}\right)=0 \quad \forall n, m .
$$

Therefore,

$$
P_{g}\left(z_{1} \ldots z_{g-1}\right) \sim \prod_{n=1}^{g} \prod_{m=1}^{g-1}\left(p_{n}-z_{m}\right)\left(q_{n}-z_{m}\right) .
$$

This product is the polynomial of the power $2 g$ with respect to each $z_{m}$, and this contradicts with the structure of $P_{g}$, therefore $P_{g} \equiv 0$. Hence we prove

$$
H^{(g)}\left(\left\{\prod_{m=1}^{g-1} \sigma_{n}\left(z_{m}\right)^{-1}\right\}_{n=1}^{g}\right)=0 \quad \forall z_{1}, \ldots, z_{g-1} .
$$

Backward, consider equation $H^{(g)}\left(\left\{f_{n}\right\}_{n=1}^{g}\right)=0$. Since $H^{(g)}$ is the first power polynomial with respect to each $f_{n}$, solution of $H^{(g)}=0$ is a rational $\mathbb{C}^{g-1}$ variety, so as $f_{n}=\prod_{m=1}^{g-1} \sigma_{n}\left(z_{m}\right)^{-1}$.

Proposition .2 (Casoratti determinant representation). The function $H^{(g)}$ may be presented as the determinant

$$
H^{(g)}\left(\left\{f_{n}\right\}_{n=1}^{g}\right)=\frac{\operatorname{det}\left|q_{j}^{i-1}-f_{j} p_{j}^{i-1}\right|_{i, j=1}^{g}}{\prod_{i>j}\left(q_{i}-q_{j}\right)} .
$$

Proof. Evidently, zeros of the right-hand side of (A.16) correspond to the case when the columns of the matrix $\left|q_{j}^{i-1}-f_{j} p_{j}^{i-1}\right|_{i, j=1}^{g}$ are linearly dependent, that is, there exists $c_{i}, i=1, \ldots, g$ such that

$$
\sum_{i=1}^{g} c_{i} q_{j}^{i-1}=f_{j} \sum_{i=1}^{g} c_{i} p_{j}^{i-1} .
$$

Let $\sum_{i=1}^{g} c_{i} z^{i-1}=\prod_{m=1}^{g-1}\left(z-z_{m}\right)$, then the determinant is zero if and only if

$$
f_{j}=\prod_{m=1}^{g-1} \frac{q_{j}-z_{m}}{p_{j}-z_{m}}=\prod_{m=1}^{g-1} \sigma_{j}\left(z_{m}\right)^{-1} .
$$

Therefore, due to Proposition 1 the determinant is proportional to $H^{(g)}$. The denominator in the right-hand side of (A.16) is the subject of normalization when $f_{j}=0$. 
Proposition .3 (Theta-functions and prime forms). The function $H^{(g)}$ has the following identity:

$$
\begin{aligned}
H^{(g)}\left(\left\{f_{n}=\sigma_{n}(\lambda) \prod_{m=1}^{g} \sigma_{n}\left(z_{m}\right)^{-1}\right\}_{n=1}^{g}\right) \\
=\left(\prod_{m=1}^{g} \frac{\lambda-z_{m}}{\lambda-q_{m}}\right) H^{(g)}\left(\left\{f_{n}=\prod_{m=1}^{g} \sigma_{n}\left(z_{m}\right)^{-1}\right\}_{n=1}^{g}\right) .
\end{aligned}
$$

Proof. A simple application of the recursion relation (A.9) to $H^{(g+1)}(\{\cdots\})=0$, where $p_{g+1}=\infty$ and $q_{g+1}=\lambda$.

\section{.2. The Fay identity}

Proposition .4 (Fay identity).

$$
\begin{gathered}
(A-D)(C-B) H^{(g)}\left(\left\{f_{n} \frac{\sigma_{n}(A)}{\sigma_{n}(B)}\right\}_{n=1}^{g}\right) H^{(g)}\left(\left\{f_{n} \frac{\sigma_{n}(C)}{\sigma_{n}(D)}\right\}_{n=1}^{g}\right) \\
+(A-B)(D-C) H^{(g)}\left(\left\{f_{n} \frac{\sigma_{n}(A)}{\sigma_{n}(D)}\right\}_{n=1}^{g}\right) H^{(g)}\left(\left\{f_{n} \frac{\sigma_{n}(C)}{\sigma_{n}(B)}\right\}_{n=1}^{g}\right) \\
=(A-C)(D-B) H^{(g)}\left(\left\{f_{n}\right\}_{n=1}^{g}\right) H^{(g)}\left(\left\{f_{n} \frac{\sigma_{n}(A) \sigma_{n}(C)}{\sigma_{n}(B) \sigma_{n}(D)}\right\}_{n=1}^{g}\right) .
\end{gathered}
$$

Proof. Let LHS $\left(\left\{f_{n}\right\}_{n=1}^{g}\right)$ be the left-hand side of (A.20). It is a second-order polynomial with respect to each of $f_{1}, \ldots, f_{g}$. Using Proposition .3 , it may be shown that

$$
\begin{aligned}
\operatorname{LHS}\left(\left\{f_{n}\right.\right. & \left.\left.=\frac{\sigma_{n}(B) \sigma_{n}(D)}{\sigma_{n}(A) \sigma_{n}(C)} \prod_{m=1}^{g-1} \sigma_{n}\left(z_{m}\right)^{-1}\right\}_{n=1}^{g}\right) \\
& =\operatorname{LHS}\left(\left\{f_{n}=\prod_{m=1}^{g-1} \sigma_{n}\left(z_{m}\right)^{-1}\right\}_{n=1}^{g}\right)=0
\end{aligned}
$$

for any set of $z_{1}, \ldots, z_{g-1}$. Due to Proposition .1, it means unambiguously

$$
\operatorname{LHS}\left(\left\{f_{n}\right\}\right)=\text { const } H^{(g)}\left(\left\{f_{n}\right\}_{n=1}^{g}\right) H^{(g)}\left(\left\{f_{n} \frac{\sigma_{n}(A) \sigma_{n}(C)}{\sigma_{n}(B) \sigma_{n}(D)}\right\}_{n=1}^{g}\right) .
$$

Taking, for example, $f_{n}=\left(\sigma_{n}(D) / \sigma_{n}(A)\right) \prod_{m=1}^{g-1} \sigma_{n}\left(z_{m}\right)^{-1}$, we fix the constant and obtain finally the given form (A.20) of the coefficients.

.3. Application to the relativistic Toda chain. Let all parameters $\left\{p_{n}, q_{n}\right\}_{n=1}^{\infty}$ be related by

$$
p_{n}+q_{n}=1-\kappa^{N} p_{n} q_{n} \quad \forall n
$$

so that

$$
p_{n}=\frac{1}{\Delta_{\phi_{n}}}, \quad q_{n}=\frac{1}{\Delta_{\phi_{n}}^{*}}
$$


in the notations of (2.30). Note,

$$
\sigma_{n}\left(-\frac{1}{\kappa^{N}}\right)=\sigma_{n}(0) \sigma_{n}(1),
$$

and in the $\Delta, \Delta^{*}$-notations $(5.24)$

$$
\mathbf{e}^{2 i \phi_{n}}=\sigma_{n}(0)^{-1}, \quad s_{n}(1)=\sigma_{n}(1), \quad s_{n}\left(\Delta_{m}^{*}\right)=\sigma_{n}\left(q_{m}\right), \quad s_{n}\left(\Delta_{m}\right)=\sigma_{n}\left(p_{m}\right) .
$$

Besides, when $\left\{p_{n}, q_{n}\right\}$ obey (A.24), we have $H^{(g)} \equiv h^{(g)}$ (see (5.23)).

Taking $z_{1}=\cdots=z_{m}=1$ and $z_{m+1}=\cdots=z_{g-1}=\infty$ in Proposition .1, we obtain

$$
h^{(g)}\left(\left\{\mathbf{e}^{2 i m \phi_{n}}\right\}_{n=1}^{g}\right)=0, \quad m=0, \ldots, g-1 .
$$

Consider discrete equations (5.18) and (5.20) with $m \in \mathbb{Z}$ and $n \in \mathbb{Z}_{+}$. Omitting the index $n$ in $\tau$-functions, we rewrite these relations as follows:

$$
\begin{gathered}
\left(\delta_{g} \delta_{g}^{*} \boldsymbol{\tau}_{m}^{\prime} \boldsymbol{\tau}_{m} \theta_{m-1}\right)^{N}=\left(\delta_{g} \boldsymbol{\tau}_{m-1}^{\prime} \boldsymbol{\tau}_{m} \theta_{m}\right)^{N}+\left(\delta_{g}^{*} \boldsymbol{\tau}_{m+1}^{\prime} \boldsymbol{\tau}_{m-1} \theta_{m-1}\right)^{N}+\left(\kappa \boldsymbol{\tau}_{m}^{\prime} \boldsymbol{\tau}_{m-1} \theta_{m}\right)^{N} \\
\left(\theta_{m}^{\prime}\right)^{N}=\left(\frac{w_{\phi}}{\boldsymbol{\tau}_{m-1}}\right)^{N}\left(\left(\theta_{m} \boldsymbol{\tau}_{m-1}^{\prime}\right)^{N}-\left(\delta_{g}^{*} \theta_{m-1} \boldsymbol{\tau}_{m}^{\prime}\right)^{N}\right)
\end{gathered}
$$

Proposition .5. Let

$$
\begin{aligned}
& \left(\tau_{m}\right)^{N}=H^{(g-1)}\left(\left\{f_{n} \mathrm{e}^{2 i \phi_{n} m}\right\}_{n=1}^{g-1}\right), \\
& \left(\theta_{m}\right)^{N}=H^{(g-1)}\left(\left\{f_{n} \mathrm{e}^{2 i \phi_{n} m} \sigma_{n}(1)\right\}_{n=1}^{g-1}\right) .
\end{aligned}
$$

Then the complete solution of (A.28) and (A.29) are

$$
\begin{aligned}
& \left(\tau_{m}^{\prime}\right)^{N}=H^{(g)}\left(\left\{f_{n} \mathrm{e}^{2 i \phi_{n} m}\right\}_{n=1}^{g}\right), \\
& \left(\theta_{m}^{\prime}\right)^{N}=H^{(g-1)}\left(\left\{f_{n} \mathrm{e}^{2 i \phi_{n} m} \sigma_{n}(1)\right\}_{n=1}^{g}\right),
\end{aligned}
$$

where $\mathbf{e}^{2 i \phi_{g}}=\left(\delta_{g} / \delta_{g}^{*}\right)^{N}$, all $f_{1}, \ldots, f_{g-1}$ are the same in (A.30) and (A.31), and $f_{g}$ is arbitrary extra complex number.

Proof. The index $m$ enters (A.30) as a scale of $f_{n}$, so later we may consider only $m=0$.

Note that due to (A.9) and the fact $\sigma_{g}\left(q_{g}\right)^{-1}=0$, we have

$$
H^{(g)}\left(\left\{f_{n} \sigma_{n}\left(q_{g}\right)^{-1}\right\}_{n=1}^{g}\right) \equiv H^{(g-1)}\left(\left\{f_{n}\right\}_{n=1}^{g-1}\right) .
$$

For given set of $\left\{f_{n}\right\}_{n=1}^{g}$ consider two copies of Fay's identity: the first one with

$$
A=0, \quad B=q_{g}, \quad C=1, \quad D=-\frac{1}{\kappa^{N}},
$$

and the second one with

$$
A=\infty, \quad B=q_{g}, \quad C=-\frac{1}{\kappa^{N}}, \quad D=1 .
$$


Condition (A.23) is implied. Excluding $H^{(g)}\left(\left\{f_{n} \sigma_{n}(1)^{-1}\right\}_{n=1}^{g}\right)$ from both these equations, we obtain exactly (A.28) with the identification (A.30), (A.31). Equation (A.29) corresponds to the Fay identity with

$$
A=0, \quad B=q_{g}, \quad C=1, \quad D=\infty .
$$

Note in conclusion, evolution equation (6.39) corresponds to the Fay identity with

$$
A=\infty, \quad B=0, \quad C=-\frac{1}{\kappa^{N}}, \quad D=1 .
$$

ACKNOWLEDGments. The authors are grateful to R. Baxter, V. Bazhanov, V. Mangazeev, G. Pronko, E. Sklyanin, A. Belavin, Yu. Stroganov, A. Isaev, G. von Gehlen, V. Tarasov, P. Kulish, F. Smirnov, and R. Kashaev for useful discussions and comments. This work was supported in part by the grant INTAS OPEN 00-00055. The work of Pakuliak was supported by the Russian Foundation for Basic Research, RFBR 00-0216477 and the grant for support of scientific schools RFBR 00-15-96557, the work of Sergeev was supported by the grant RFBR 01-01-00201.

\section{REFERENCES}

[1] R. J. Baxter, J. H. H. Perk, and H. Au-Yang, New solutions of the star-triangle relations for the chiral Potts model, Phys. Lett. A 128 (1988), no. 3-4, 138-142.

[2] V. Bazhanov, A. Bobenko, and N. Reshetikhin, Quantum discrete sine-Gordon model at roots of 1: integrable quantum system on the integrable classical background, Comm. Math. Phys. 175 (1996), no. 2, 377-400.

[3] V. V. Bazhanov and N. Yu. Reshetikhin, Remarks on the quantum dilogarithm, J. Phys. A 28 (1995), no. 8, 2217-2226.

[4] V. V. Bazhanov and Yu. G. Stroganov, Chiral Potts model as a descendant of the six-vertex model, J. Statist. Phys. 59 (1990), no. 3-4, 799-817.

[5] L. D. Faddeev, Discrete Heisenberg-Weyl group and modular group, Lett. Math. Phys. 34 (1995), no. 3, 249-254.

[6]___ Modular double of a quantum group, Conférence Moshé Flato 1999, Vol. I (Dijon), Math. Phys. Stud., vol. 21, Kluwer Academic Publishers, Dordrecht, 2000, pp. 149156.

[7] L. D. Faddeev and R. M. Kashaev, Quantum dilogarithm, Modern Phys. Lett. A 9 (1994), no. 5, 427-434.

[8] L. D. Faddeev, R. M. Kashaev, and A. Yu. Volkov, Strongly coupled quantum discrete Liouville theory. I. Algebraic approach and duality, Comm. Math. Phys. 219 (2001), no. 1, 199-219.

[9] S. Kharchev and D. Lebedev, Integral representation for the eigenfunctions of a quantum periodic Toda chain, Lett. Math. Phys. 50 (1999), no. 1, 53-77.

[10] S. Kharchev, D. Lebedev, and M. Semenov-Tian-Shansky, Unitary representations of $U_{\mathcal{q}}(\mathfrak{s}[(2, \mathbb{R}))$, the modular double and the multiparticle q-deformed Toda chains, Comm. Math. Phys. 225 (2002), no. 3, 573-609.

[11] A. Kundu, Generation of a quantum integrable class of discrete-time or relativistic periodic Toda chains, Phys. Lett. A 190 (1994), no. 1, 79-84.

[12] V. B. Kuznetsov, M. Salerno, and E. K. Sklyanin, Quantum Bäcklund transformation for the integrable DST model, J. Phys. A 33 (2000), no. 1, 171-189.

[13] V. B. Kuznetsov and E. K. Sklyanin, On Bäcklund transformations for many-body systems, J. Phys. A 31 (1998), no. 9, 2241-2251.

[14] V. B. Kuznetsov and A. V. Tsiganov, Separation of variables for the quantum relativistic Toda lattices, J. Math. Sci. 80 (1994), no. 3, 1802-1810. 
[15] S. Pakuliak and S. Sergeev, Relativistic Toda chain at root of unity, preprint ITEP-TH-86/00, 2001 http://arxiv.org/abs/nlin.SI/0101024.

[16] _ Relativistic Toda chain at root of unity II. Modified Q-operator, preprint MPI 200165, 2001 http://arxiv.org/abs/nlin.SI/0107062.

[17] _ Relativistic Toda chain at root of unity III. Relativistic Toda chain hierarhy, preprint MPI 2001-66, 2001 http://arxiv.org/abs/nlin.SI/0107063.

[18] G. Pronko and S. Sergeev, Relativistic Toda chain, to appear in J. Appl. Math.

[19] S. N. M. Ruijsenaars, Relativistic Toda systems, Comm. Math. Phys. 133 (1990), no. 2, 217-247.

[20] S. M. Sergeev, Quantum $2+1$ evolution model, J. Phys. A 32 (1999), no. 30, 5693-5714.

[21] Auxiliary transfer matrices for three-dimensional integrable models, Teoret. Mat. Fiz. 124 (2000), no. 3, 391-409.

[22] - On exact solution of a classical 3D integrable model, J. Nonlinear Math. Phys. 7 (2000), no. 1, 57-72.

[23] _ Quantum matrices of the coefficients of a discrete linear problem, Zap. Nauchn. Sem. S.-Peterburg. Otdel. Mat. Inst. Steklov. (POMI) 269 (2000), no. Vopr. Kvant. Teor. Polya i Stat. Fiz. 16, 292-307, 370-371.

[24] _ Solitons in a 3d integrable model, Phys. Lett. A 265 (2000), no. 5-6, 364-368.

[25] S. M. Sergeev, V. V. Mangazeev, and Yu. G. Stroganov, The vertex formulation of the Bazhanov-Baxter model, J. Statist. Phys. 82 (1996), no. 1-2, 31-49.

[26] E. K. Sklyanin, The quantum Toda chain, Nonlinear Equations in Classical and Quantum Field Theory (Meudon/Paris, 1983/1984), Lecture Notes in Phys., vol. 226, Springer, Berlin, 1985, pp. 196-233.

[27] _ Functional Bethe ansatz, Integrable and Superintegrable Systems (B. A. Kupershmidt, ed.), World Scientific Publishing, New Jersey, 1990, pp. 8-33.

[28] _ _ Quantum inverse scattering method. Selected topics, Quantum Group and Quantum Integrable Systems (Mo-Lin Ge, ed.), Nankai Lectures in Mathematical Physics, World Scientific Publishing, New Jersey, 1992, pp. 63-97.

[29] _ Separation of variables - new trends, Progr. Theoret. Phys. Suppl. (1995), no. 118, 35-60.

[30] _ Bäcklund transformations and Baxter's Q-operator, Integrable Systems: From Classical to Quantum (Montréal, QC, 1999), Lecture Notes, vol. 26, American Mathematical Society, Rhode Island, 2000, pp. 227-250.

[31] Yu. G. Stroganov, private communications.

[32] Yu. B. Suris, Discrete time generalized Toda lattices: complete integrability and relation with relativistic Toda lattices, Phys. Lett. A 145 (1990), no. 2-3, 113-119.

[33] V. Tarasov, Cyclic monodromy matrices for $\operatorname{sl}(n)$ trigonometric $R$-matrices, Comm. Math. Phys. 158 (1993), no. 3, 459-483.

STANiSLAV PAKULIAK: BOgOliUbOV LABORATORY OF THEORETICAL PHYSiCS, JOINT INSTITUTE FOR NuClEAR RESEARCH, DUBNA 141980, MOSCOW, RuSSIA

E-mail address: paku1iak@thsun1.jinr.ru

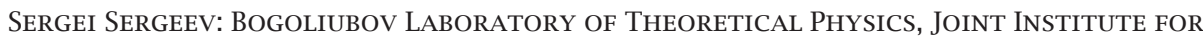
NuCLEAR RESEARCH, DubNA 141980, MOSCOW, RuSSIA

E-mail address: sergeev@thsun1.jinr.ru 


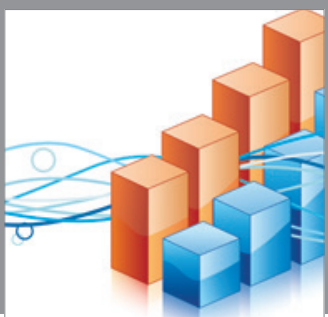

Advances in

Operations Research

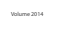

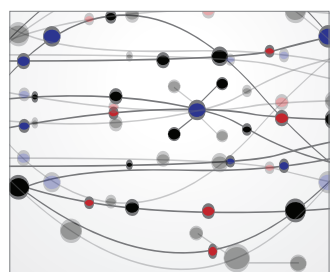

\section{The Scientific} World Journal
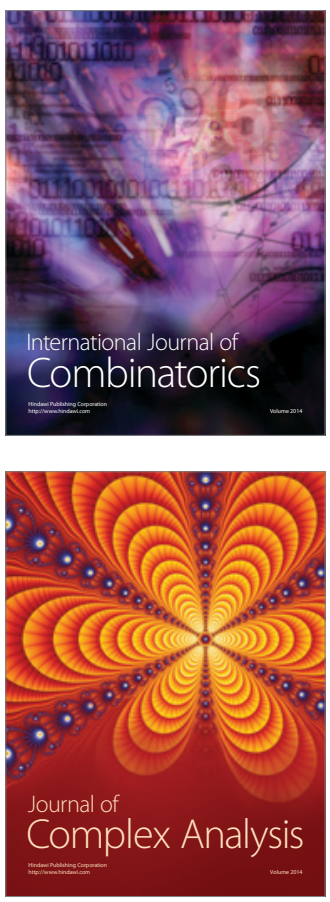

International Journal of

Mathematics and

Mathematical

Sciences
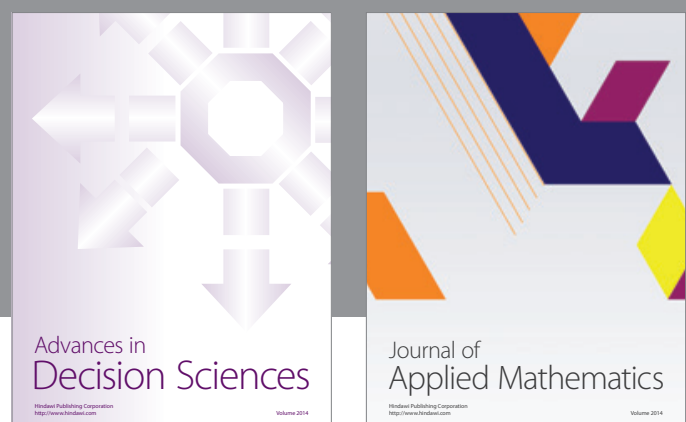

Journal of

Applied Mathematics
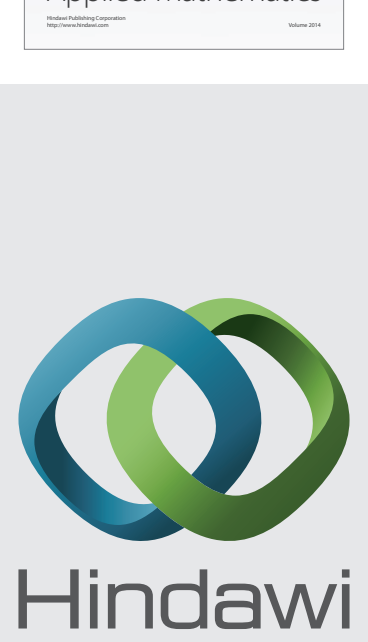

Submit your manuscripts at http://www.hindawi.com
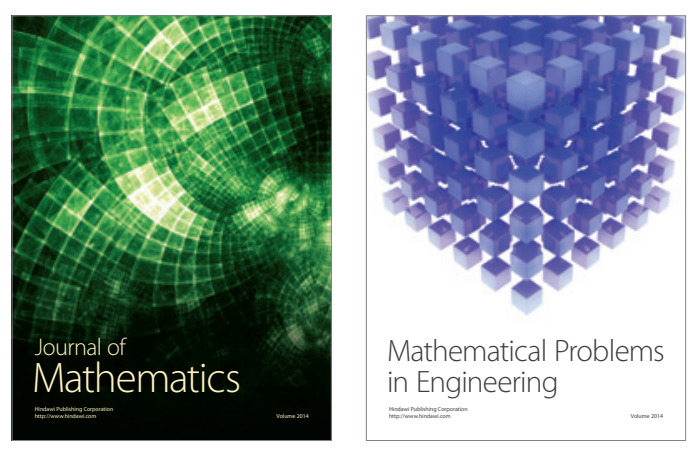

Mathematical Problems in Engineering
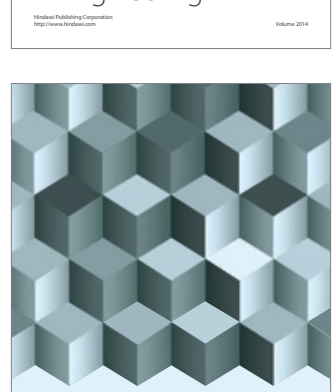

Journal of

Function Spaces
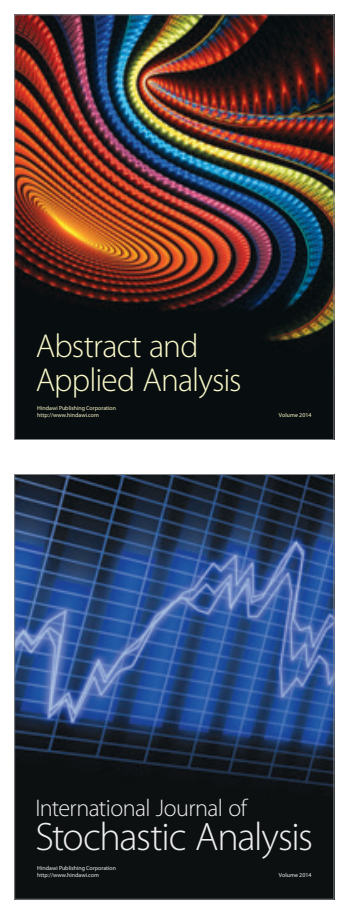

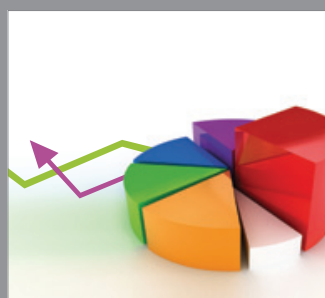

ournal of

Probability and Statistics

Promensencen
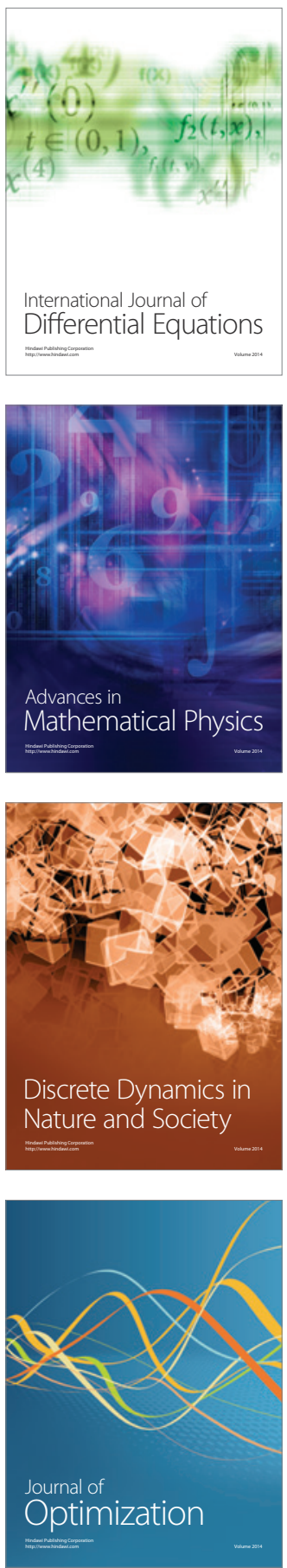